\section{Language teaching}

doi:10.1017/S0261444805212995

\section{5-206 Chan, Tun-Pei \& Hsien-Chin Liou (National Tsing Hua U, Taiwan, China), Effects of web-based concordancing instruction on EFL students' learning of verb-noun collocations. Computer Assisted Language Learning (CALL) (London, UK) 18.3 (2005), 231-251.}

This study investigates the influence of using five webbased practice units on English verb-noun collocations with the design of a web-based Chinese-English bilingual concordancer (keyword retrieval program) on collocation learning. Thirty-two college EFL students participated by taking a pre-test and two post-tests, and responding to a background questionnaire and an evaluation questionnaire. Results indicated that learners made significant collocation improvement immediately after the online practice but regressed later. Yet, the final performance was still better than students' entry level. Different verb-noun collocation types and learners with different prior collocation knowledge were found to be not equally receptive to the practice effects. Both the online instructional units and the concordancer were acceptable to most participants.

05-207 Chang, Mei-Mei (National Pingtung U of Science and Technology, Taiwan, China),

Applying self-regulated learning strategies in a web-based instruction - an investigation of motivation perception. Computer Assisted Language Learning (CALL) (London, UK) 18.3 (2005), 217-230.

The purpose of this study was to examine the effect of self-regulated learning strategies on learners' perception of motivation within web-based instruction. In this study, self-regulated learning strategies, which were intended to assist students to self-observe and self-evaluate their effectiveness, were incorporated into a one-semester web-based course to help students improve their learning motivation. Research results revealed that students' motivation perception benefited from the web-based instruction with self-regulated learning strategies. Students learning within a webbased environment with self-regulated learning strategies became more responsible for their own learning, more intrinsically orientated and more challengeable. They tended to value the learning material more and became more confident in course understanding and class performance.

05-208 Coleman, James A. (The Open U, UK; J.A.Coleman@open.ac.uk), CALL from the margins: effective dissemination of CALL research and good practices. ReCALL (Cambridge, UK) 17.1 (2005), 18-31.
EUROCALL's 2004 conference in Vienna offered to both new members from Eastern Europe and established members from the West an opportunity to review the relationship between computer-assisted language learning (CALL) and language teaching in general. CALL is defined as an 'academic field that explores the role of information and communication technologies in language learning and teaching'. CALL practitioners and researchers have long been aware of the importance of recognition within the broader discipline of language learning and teaching, as the joint EUROCALL/CALICO/IALL Research Policy Statement (EUROCALL 1999) noted. Yet CALL remains marginalised in several ways, which this article explores. In seeking to promote more effective dissemination of good teaching practices and especially of research in CALL, the article evokes the UK's role in introducing Quality Assurance (QA) to higher education teaching and research - a trend which the Bologna Process will intensify throughout Europe. The author draws on his current role as language research coordinator at the UK's Open University, and on experience as a QA insider in both teaching and research, to analyse successes and failures in dissemination of both research and good teaching/learning practices. $\mathrm{He}$ proposes strategies for moving CALL from the margins towards the centre of language learning and provides an overview of some key journals and conferences in the domain.

\section{5-209 Eslami-Rasekh, Zohreh (Texas A and} M U, USA; zeslami@coe.tamu.edu), Raising the pragmatic awareness of language learners. ELT Journal (Oxford, UK) $\mathbf{5 9 . 3 ~ ( 2 0 0 5 ) , ~ 1 9 9 - 2 0 8 . ~}$

The development of pragmatic and sociolinguistic rules of language use is important for language learners. It is necessary to understand and create language that is appropriate to the situations in which one is functioning, because failure to do so may cause users to miss key points that are being communicated or to have their messages misunderstood. Worse yet is the possibility of a total communication breakdown and the stereotypical labelling of second language users as people who are insensitive, rude, or inept (Thomas 1983). In this paper I will discuss different approaches to teaching pragmatics and, based on my teaching experiences, I will provide some strategies that can be used to raise the pragmatic awareness of English language learners (ELLs).

05-210 Foley, J. (SEAMEO RELC, Singapore; jfoley@relc.org.sg), English in ... Thailand. RELC Journal (Thousand Oaks, CA, USA) 36.2 (2005), 223-234. 
This account of English in the formal education system in Thailand traces briefly its historical background and then looks at the teaching of English today. The country itself has quite a complex sociolinguistic context and in order to understand the teaching and learning of English - one must understand the basic tenets of the culture. From this cross-cultural perspective, we can then understand some of the new initiatives that are presently being taken in English Language Teaching in Thailand.

05-211 Frankenberg-Garcia, Ana (ISLA, Lisbon, Portugal; ana.frankenberg@sapo.pt), Pedagogical uses of monolingual and parallel concordances. ELT Journal (Oxford, UK) 59.3 (2005), 189-198.

This paper discusses the use of concordances in the classroom, with particular reference to the pedagogical implications of the differences between parallel and monolingual concordances. Examples are given of using the two kinds of concordances in activities that involve language production, reception, correction, and testing. It is concluded that monolingual and parallel concordances have non-conflicting, complementary roles to play.

\section{5-212 Hansson, Thomas ( $U$ of Southern Denmark, Denmark), English as a second language on a virtual platform - tradition and innovation in a new medium. Computer Assisted Language Learning (CALL) (London, UK) 18.1/2 (2005), 63-79.}

A pilot study at a local school explores a virtual world during English lessons. The objective of applying a Vygotskian experimental design to the study is to investigate the potential of software, interaction and integration related to problem-solving defined as text composition in a foreign language. Focus of research and practices is on the development of a virtual didactic, more specifically text interaction by a virtual game in the classroom and face-to-face dialogue by direct speech-dialogue in the computer room. The two-fold objectives cover understanding an integrated didactic on English and computer efficacy. By investigating the design of a combined virtual and physical learning environment we describe how the video-game generation operates (in) a social system of peers as they develop their computer skills and text composing ability.

\section{5-213 Hu, Guangwei (Nanyang Technological $U$, Singapore), Using peer review with Chinese ESL student writers. Language Teaching Research (London, UK) 9.3 (2005), 321-342.}

Peer review has a prominent place in process-oriented writing instruction. In this paper, I share my developing use of peer review in an academic writing course for Chinese ESL learners. After reviewing the relevant literature, I describe the context in which I implemented peer review and conducted practitioner research into its productive use. Next, I present and discuss the training activities and follow-ups I used to prepare my students for effective peer review. The various instructional practices have been experimented with and refined in a 3-year action research effort to best facilitate my students' acquisition of academic writing skills and hence enhance the quality of classroom life. In conclusion I summarise student performance in my most recent writing class and consider several instructional changes that are likely to help students understand better and thus benefit more from peer review.

05-214 Jung, Udo O. H. (Bonn, Germany; hmejung@gmx.de), CALL: past, present and future - a bibliometric approach. ReCALL (Cambridge, UK) 17.1 (2005), 4-17.

A bibliometric approach has been used not only to sketch out the development of CALL (computer assisted language learning) during the last 25 years, but also to assess the contribution of educational technology to 21st century foreign-language teaching and learning. This study is based on the six instalments of the author's International (and multilingual) Bibliography of Computer-Assisted Language Learning and the 5,301 its entries. The once text-based bibliography has been transformed into a searchable database. Since index terms to describe both the contents and the nature of individual publications have been attached to the bibliographic data, it is now possible to query whether the 25,000 descriptors cluster around certain topics and to depict developments chronologically. The statistical evaluation of a large corpus also avoids the pitfalls of selective interpretation. Recent controversies about the chronologisation of CALL events as well as the internal consistency of such time chunks are addressed. The data suggest that the online/offline divide occurs around 1993 and that the latest additions to the foreign language teacher's tool box - from emails to voicechats overcome the language acquisition/language learning barrier. New and student-oriented forms of dealing with foreign language learning come to the fore. This has induced some researchers to concentrate on events where conversation breaks down, because learners must then ask for modified input or negotiate the meanings of lexical items. Such a strategy promises success in instructed second-language acquisition. It is suggested, however, that the foreign language teacher's intervention is a necessary complement to second-language developmental processes.

\footnotetext{
05-215 Kaltenböck, Gunther \& Barbara MehImauer-Larcher ( $U$ of Vienna, Austria; gunther.kaltenboeck@univie.ac.at), Computer corpora and the language classroom: on the potential and limitations of computer corpora in language teaching. ReCALL (UK) 17.1 (2005), 65-84.
} 
With computer corpora firmly established as research tools in linguistics, their application for language teaching purposes is also increasingly advocated to the extent that corpus-based language teaching has even been praised as the new revolution in language teaching. This article takes a more critical view and examines some of the potential as well as the limitations of computer corpora in the language classroom, providing practical examples from the British Component of the International Corpus of English. It is argued that only a balanced view, which takes into account both the strengths and weaknesses of computer corpora for language teaching, can ensure their successful integration into the language classroom. The discussion first focuses on the limitations of corpus data, which are identified as 'externalised', as opposed to 'internalised' language, lacking contextual, as opposed to co-textual, information. On the other hand, computer corpora provide access to information not easily available from other sources, viz. information on frequency of occurrence in various text types, and co-occurrence patterns, e.g. collocation, colligation, semantic prosody). This information, however, also has to be seen in the light of the more general questions of how representative corpus results are and to what extent they are generalisable. The article concludes with a discussion of pedagogical implications of the use of computer corpora, especially with regard to their application as tools for exploratory/discovery learning and as means for promoting learner autonomy.

\section{5-216 Lasagabaster, David \& Juan M. Sierra (U del País Vasco-Euskal Herriko Unibertsitatea, Spain), Error correction: students' versus teachers' perceptions. Language Awareness (Clevedon, UK) 14.2/3 (2005), 112-128.}

Few issues in second language teaching have generated as much controversy as that of error correction. In fact, one of the most discouraging experiences of L2 teachers is correcting errors especially those that recur in their students' production. A possible explanation may be the mismatch between what teachers and students consider to be effective feedback on error correction. Although much has been published on error taxonomies, detection, analysis and evaluation, there is a dearth of research studies comparing teachers' and students' perceptions. This article endeavours to help fill this void. The study involved 21 informants. Eleven of them were undergraduate students who had no teaching experience or qualifications, whereas the other 10 were qualified teachers of English as a foreign language with between three and 13 years' experience. After watching an excerpt from a commercially produced teaching video twice, the participants were asked to detect the error-correction moves made by the teacher, classify them, judge their efficiency and record their opinions individually and in groups. The results indicated that a significant percentage of the teacher's error-correction moves went unnoticed. Teachers and students agreed that the most efficient corrections occurred when more time, longer explanations, and use of different correction strategies were utilised.

\section{5-217 Liu, Gi-Zen (National Cheng Kung U,} Taiwan, China; gizenliu@gmail.com), The trend and challenge for teaching EFL at Taiwanese universities. RELC Journal (Thousand Oaks, CA, USA) 36.2 (2005), 211-221.

The author first talks about the current situation of teaching EFL in higher education institutions in Taiwan, and indicates that the Communicative Language Teaching (CLT) Approach has been the most popular language teaching approach lately. $\mathrm{He}$ then addresses the hybrid course approach which seems to be a trend as well as an appropriate way for teaching English. Despite the popularity of CLT, the situation regarding implementation of CLT in Taiwanese higher education institutions is complex. The author discusses the background of Taiwanese EFL instructors, the contradictory findings about EFL learners' preferences and needs, and the existing English learning problem among college students in the effort to provide a solution. In addition, he emphasises the implementation and application of the Internet in Taiwan for facilitating e-learning and English learning with the adaptation of instructor's role and student's role when using CLT at Taiwanese universities in the Internet age.

05-218 Lundy, Garvey F. (Pennsylvania U, USA; garvey@pop.upenn.edu), School resistance in American high schools: the role of race and gender in oppositional culture theory. Evaluation \& Research in Education (Clevedon, UK) 17.1 (2003), 6-30.

Oppositional culture theory has been primarily used to explain race and ethnic differences in education. It argues that members of involuntary minority groups tend to underachieve for fear of being sanctioned by their peers. The underlying assumption, then, is that academic success harms peer relationships for involuntary minorities more than it does for other groups. Prior tests based on survey data fail to support the theory. Using the National Education Longitudinal Study (NELS), Race/ethnic and gender differences are examined here with respect to the two main components of oppositional culture theory: peer relations and school resistance. Like prior survey analyses, no support was found for the thesis that oppositional culture accounts for race/ethnic differences in school achievement. However, oppositional culture does appear to play a key role in explaining why male students tend to receive lower grades despite standardised test scores that equal or exceed the scores of female students. On the basis of several measures in the NELS, antistudious attitudes and behaviours are found to be more prevalent among males than females. Future researchers should be more sensitive to this gender aspect of school culture. 
05-219 Nemtchinova, Ekaterina (Seattle Pacific U, USA; katya@spu.edu), Host teachers' evaluations of non-native-English-speaking teacher trainees: a perspective from the classroom. TESOL Quarterly (Alexandria, VA, USA) 39.2, 235-262.

This article discusses the results of a survey conducted to explore how students and host teachers perceive the strengths of non-native-English-speaking (NNES) teacher trainees based on their classroom practice. Responding to a questionnaire evaluating the NNES English-as-a-second language (ESL) teacher trainees in their classrooms, host teachers named the following as their strengths: teaching ability, professional skills, the grammaticality and idiomaticity of their English, and the multilingual and multicultural resources that they bring. Host teachers noted that NNES teacher trainees understood their students' learning problems and concerns, often served as role models for ESL students, and enriched the classroom experience. A small percentage of host teachers were dissatisfied with their trainee's performance because they felt the trainee had inadequate command of English. The results of the survey suggest that NNES teacher trainees are generally perceived as capable of delivering efficient instruction and treated as a welcome addition to the ESL practicum classroom.

\section{5-220 Nickerson, Catherine, Marinel Gerritsen \& Frank v. Meurs (Radboud U, Nijmegen, the Netherlands; c.nickerson@let.ru.nl), \\ Raising student awareness of the use of English for specific business purposes in the European context: a staff-student project. English for \\ Specific Purposes (Amsterdam, the Netherlands)} 24.3 (2005), 333-345.

This paper reports on a large-scale staff-student project focussing on the use of English for Specific Business Purposes in a number of promotional genres (TV commercials, annual reports, corporate websites, print advertising) within several of the EU member states: Belgium, the Netherlands, France, Germany and Spain. The project as a whole aims to identify where and to what extent English is used and also to investigate the consequences of including English in promotional genres where corporations are presumably intending to reach and persuade audiences in non-English-speaking countries. Focus is therefore on the occurrence of English, the attitudes of consumers to the use of that English, and whether or not they are able to understand it. This paper reports in detail on a survey of the use of English in print advertising in the July 2003 editions of glossy magazines aimed at young women in the Netherlands, Germany and Spain, together with an experimental investigation of the attitudes to and comprehension of English, based on data collected from between 43 and 50 respondents in each of the three countries. The project has been invaluable in raising student awareness of the way in which English is pre- sent in the world around them and, more specifically, that English is increasingly becoming an intrinsic part of a number of highly visible and highly accessible promotional genres.

\section{5-221 Palfreyman, David (Zayed U, Dubai, $U A E)$, Othering in an English Language Program. TESOL Quarterly (Alexandria, VA, USA) 39.2,} 211-234.

In this article, I discuss how the administrators and local teachers in a Turkish university English language centre perceive others in the institution. I present interview data to illustrate processes of OTHERING, whereby a group constructs a shared, Us-Them representation of another group. The data show that administrators and local teachers view students and each other in terms of difference from themselves. In constructing such representations, they draw on local and wider discourses about learning, social order, national and institutional characteristics, class, and gender. Interrogating biases and developing a deeper understanding of Othering in TESOL contexts can help English language educators to develop appropriate and authentic pedagogies and curricula for local contexts in an increasingly globalised world.

05-222 Sonck, Gerda (U Libre de Bruxelles, Belgium), Language of instruction and instructed languages in Mauritius. Journal of Multilingual and Multicultural Development (Clevedon, UK) 26.1 (2005), 37-51.

Mauritius is a multilingual country with English, French and Creole as the main languages, and several ancestral languages which are mainly used for religious ceremonies. Most children speak Creole at home and learn English, French and one ancestral language in the first year of primary school. The educational dropout rate is $40-50 \%$ after primary school. It is argued that the use of three languages in primary schooling places too great a cognitive burden on children. Language policy in education needs to be revised on pedagogical and scientific rather than political grounds. It is recommended that languages be introduced into the curriculum at different points in time.

05-223 Svalberg, Agneta M. L. (U of Leicester, UK), Consciousness-raising activities in some Lebanese English language classrooms: teacher perceptions and learner engagement. Language Awareness (Clevedon, UK) 14.2/3 (2005), 170-193.

The article starts from the premise that approaches to the teaching of English can only be assessed in the particular settings where they may be used. Cultures of learning, teachers' and students' beliefs and prevailing material conditions will all have a bearing on how a particular approach is received. In the case study reported on here, four teachers implemented a consciousness-raising (CR) approach to grammar instruction with nearly 200 
students in years 7, 8 and 9 of a Lebanese secondary school. The teachers' and students' reactions to the particular set of activities were recorded in teacher diaries and reports and through interviews with the teachers and their coordinator, and discussions with the participating students. Both teachers and students felt generally positive towards the approach. The prevailing culture of learning, which encourages learner autonomy and an analytical approach to language, seems to have contributed. It was found, however, that in order to avoid discrepancies in the use of metalanguage and classifications and to allow insights from CR to be used in language production-for-practice, CR would need to be an integral part of the regular integrated skills syllabus.

\section{5-224 Wegerif, Rupert (Southampton U, UK;} R.B.Wegerif@soton.ac.uk), Reason and creativity in classroom dialogues. Language and Education (Clevedon, UK) 19.3 (2005), 223-237.

The development of reason has long been an important aim for education - possibly reflected in the emphasis on the importance of explicit verbal reasoning in definitions of 'Exploratory Talk'. This paper argues from transcript evidence that, while Exploratory Talk is a specific dialogical model of reason that has proved to be a useful pedagogic tool, there are educationally valuable ways of talking together that are characterised more by verbal creativity than by explicit reasoning. Close analysis of actual dialogues highlights the essential importance of verbal creativity even to the task of solving reasoning test problems in small groups. This analysis also suggests that the extent and quality of creativity found in classroom dialogues is influenced by shared ground rules. This implies the need to expand our understanding of dialogical reason to incorporate creativity and to develop dialogical models to support the stimulation and channelling of creativity in educational contexts.

\section{Language learning}

doi:10.1017/S0261444805222991

\section{5-225 Acevedo Butcher, Carmen (Sogang U, Korea), The case against the 'native speaker'. English Today (Cambridge, UK) 21.2 (2005), 13-24.}

This paper presents a discussion about the need for fairer names for speakers of English. Focus is on discussion of current popular early 3rd-millennium definitions attached to the phrase NATIVE SPEAKER (meaning 'someone born and raised speaking correct English'), including an examination of global changes across a number of languages which suggests that a profoundly changed and changing world requires radically new linguistic terms.

05-226 Barcroft, Joe \& Mitchell S. Sommers (Washington U in St. Louis, USA; barcroft@wustl.edu), Effects of acoustic variability on second language vocabulary learning. Studies in Second Language Acquisition (Cambridge, UK) 27.3 (2005), 387-414.

This study examined the effects of acoustic variability on second language vocabulary learning. English native speakers learned new words in Spanish. Exposure frequency to the words was constant. Dependent measures were accuracy and latency of picture-to-Spanish and Spanish-to-English recall. Experiment 1 compared presentation formats of neutral (conversational) voice only, three voice types, and six voice types. No significant differences emerged. Experiment 2 compared presentation formats of one speaker, three speakers and six speakers. Vocabulary learning was superior in the higher-variability conditions. Experiment 3 partially replicated Experiment 1 while rotating voice types across subjects in moderate and no-variability conditions. Vocabulary learning was superior in the higher variability conditions. These results are consistent with an exemplar-based theory of initial lexical learning and representation.

\section{5-227 Barr, David, Jonathan Leakey \&} Alexandre Ranchoux (U of Ulster, UK), Told like it is! An evaluation of an integrated oral development pilot project. Language Learning \& Technology (U of Hawaii, Manoa, USA) 9.3 (2005), 55-78.

Much established pedagogical and CALL (computerassisted language learning) research advocates an integrated constructivist approach to the use of technology in language learning. This paper reports on a pilot project delivered to first year undergraduate French students. The project aim was to deliver a blend of collaborative and individual learning through a combination of CALL programs and online activities alongside traditional faceto-face conversation classes. Using quantitative analysis of a pre- and post-test and a variety of questionnaires, this project assessed student progress in developing oral skills across two groups, one (the treatment group) using technology and the other (the comparison group) being a traditional conversation class. Each group covered the same content and underwent the same assessment procedures. In addition, through qualitative analysis measures, the project evaluated the role played by additional variables in the learning process, as well as student and staff reactions to the two approaches. The study concludes by showing that while progress was made by both groups, the progress made by those not using technology was significantly greater than that made by students using technology over a short-term study. It also highlights the need for developing pedagogy to ensure that CALL-based teaching goes beyond rehearsal activity to achieve message-orientated communication.

05-228 Belz, Julie A. (Pennsylvania State U, USA), Intercultural questioning, discovery and tension in Internet-mediated language learning 
partnerships. Language and Intercultural

Communication (Clevedon, UK) 5.1 (2005), 3-39.

This paper examines the use of questioning as an index of intercultural competence (IC) in an Internetmediated, German-American language learning partnership. Such telecollaborative, intercultural exchanges are becoming increasingly more common as a means of providing students at one location with cost-effective access to distally located representatives of the languaculture under study. Nevertheless, researchers have not paid close attention to the linguistic characterisation of the development of IC in such partnerships, despite the fact that text-based computer-mediated communication currently constitutes the primary means of telecollaborative interaction. In his well-known model of IC, Byram (1997) links the use of appropriate questioning techniques to the operation of skills of discovery, one means by which speakers in intercultural encounters may develop impressions and attitudes of one another. I examine the ways in which the number, type, chronological placement and content of the questions posed by participants each side of a transAtlantic exchange may influence the processes of impression formation in intercultural personal relationship building. Suggestions are made for ways in which research on computer-mediated relationship building in communication studies and foreign language education may enrich one another.

\section{5-229 Berry, Roger (Lingan U, Hong Kong, China), Who do they think 'we' is? Learners' awareness of personality in pedagogic grammars. Language Awareness (Clevedon, UK) 14.2/3 (2005), 84-97.}

This paper investigates L2 English learners' reactions to mixed personality in a pedagogic grammar. Personality refers to how authors represent themselves in a text and interact with their readers (e.g. personally via the use of personal pronouns or impersonally via the use of the passive, etc.); mixed personality occurs when an author switches from one option to another. In an experiment, 195 students from Hong Kong and Poland were asked to read a text in which the author switched from we to you. The expectation that this mixing would cause we to be interpreted as exclusive was not borne out; however, some students did attach negative attributes to you as a result of its proximity to we. Another finding was that awareness of the generic reference of we was higher than that for you. Writers of pedagogic grammars are encouraged to be more aware of the referential complexities of personal pronouns.

05-230 Braun, Sabine ( $U$ of Tübingen, Germany; sabine.braun@uni-tuebingen.de), From pedagogically relevant corpora to authentic language learning contents. ReCALL (Cambridge, UK) 17.1 (2005), 47-64.

The potential of corpora for language learning and teaching has been widely acknowledged, and their ready availability on the Web has facilitated access for a broad range of users, including language teachers and learners. However, the integration of corpora into general language learning and teaching practice has so far been disappointing. This paper argues that the shape of many existing corpora, designed with linguistic research goals in mind, clashes with pedagogic requirements for corpus design and use. Hence, a 'pedagogic mediation of corpora' is required (cf. Widdowson 2003). It shows that the realisation of this requirement touches on both the development of appropriate corpora and the ways in which they are exploited by learners and teachers. A small English Interview Corpus (ELISA) is used to outline possible solutions for a pedagogic mediation. The major aspect of this is the combination of two approaches to the analysis and exploitation of a pedagogically relevant corpus: a corpus-based and a discoursebased approach.

\section{5-231 Chambers, Angela (U of Limerick,} Ireland; Angela.Chambers@ul.ie), Integrating corpus consultation in language studies. Language Learning \& Technology (Hawaii, Manoa, USA) 9.2 (2005), 111-125.

The potential of corpora as a language teaching and learning resource has been evident since the 1960s, although there is little evidence available to suggest learners use such a resource to either complement dictionary consultation or as an alternative to studying grammar books. This article outlines published research on corpus consultation and describes how five secondyear undergraduates on a short course on language and technology were trained to consult corpora in languages, including French, English and Spanish, as part of their BA in languages, computing or cultural studies. The article examines the outcome of the students' corpora consultation, the problems they faced, and their evaluation of the activity that expressed satisfaction with the use of the program involved, Wordsmith Tools. It also reports on how the students appreciated access to a large number of authentic, relevant examples. In spite of the students' negative comments, such as the belief that concordancing programmes could never replace grammar books, the article concludes that corpus consultation may complement more traditional language learning and outlines how such a tool might be integrated in the classroom environment. The author suggests a need for studies of concordances prepared by the teacher that are used in sectors other than higher education.

\section{5-232 Cortés, lleana, Jesús Ramirez, María Rivera, Marta Viada \& Joan Fayer ( $U$ of Puerto} Rico, Puerto Rico), Dame un hamburger plain con ketchup y papitas. English Today (Cambridge, UK) 21.2 (2005), 35-42.

One outcome of language contact is lexical borrowing. Borrowing in Puerto Rico (for political, economic and social reasons) is evident in the influence English has had on Spanish, especially in lexical terms. This paper 
explores the impact of American English on the lexicon of Puerto Rican Spanish, specifically on vocabulary relating to food. Data were collected through participant observation in selected fast-food restaurants from different regions in Puerto Rico. An analysis of the corpus provides the basis for five categories useful in understanding the influence of English on Spanish in this domain. The study indicates that English borrowings have had a tremendous influence on the Puerto Rican lexicon, and predicts that, even though Spanish will continue to be the dominant Puerto Rican language, it will continue to change under the influence of English.

05-233 Dewaele, Jean-Marc (U of London, UK), Sociodemographic, psychological and politicocultural correlates in Flemish students' attitudes towards French and English. Journal of Multilingual and Multicultural Development (Clevedon, UK) 26.2 (2005), 118-137.

An analysis of 100 Flemish high-school students' attitudes towards French and English (both foreign languages) revealed complex links between personality factors, gender, politico-cultural identity, communicative behaviour and foreign language attitudes. Attitudes towards English were found to be much more positive than those towards French, despite the fact that the participants had enjoyed a longer and more intense formal instruction in French (it being their second language). The independent variables were found to have stronger effects for French than for English, with the exception of politico-cultural identity of the participant, which had a strong effect on attitudes towards French but not English. Overall, it seems that social factors, including exposure to the foreign languages, are linked with lower-level personality dimensions and thus shape attitudes towards these languages.

05-234 Elkhafaifi, Hussein (Washington U, USA; hme3@u.washington.edu), Listening

comprehension and anxiety in the Arabic

language classroom. The Modern Language

Journal (Malden, MA, USA) 89.2 (2005), 206-220.

Anxiety plays an important role in foreign language students' classroom performance. This study presents the results of the first empirical examination of the effect of general learning anxiety on students' achievement in an Arabic course and of listening anxiety on students' listening comprehension. The data came from two measures of anxiety and a background questionnaire administered to 233 post-secondary students of Arabic as a foreign language. Anxiety scores were correlated with final grades and listening comprehension scores. The results indicated that learning anxiety and listening anxiety are separate but related phenomena, and that both correlate negatively with achievement. The study also revealed significant negative correlations among learning anxiety, listening anxiety, and selected demographic variables. These results suggest that reducing student anxiety and providing a less stressful classroom environment might enable teachers and Arabic programs to help students improve both their listening comprehension proficiency as well as their overall course performance.

05-235 Flowerdew, Lynne (Hong Kong $U$ of Science and Technology, Hong Kong, China; Iclynne@ust.hk), Integrating traditional and critical approaches to syllabus design: the 'what', the 'how' and the 'why?'. Journal of English for Academic Purposes (Amsterdam, the Netherlands) 4.2 (2005), 135-147.

It is commonly assumed that most courses taking place in an English for Academic Purposes (EAP) setting at universities are, in the main, concerned with various EAP issues, such as listening to lectures and writing academic reports (lab reports, final-year undergraduate project reports and dissertations, etc.). This article describes a course which, while taking place in an academic setting, is designed to equip undergraduates with general skills training in English for Occupational Purposes (EOP) practices to meet students' future communication needs after graduation. EOP skills would include report writing (status, recommendation, proposal) and giving business presentations. A main feature of this EOP-oriented course is that it does not rely on a single approach to syllabus design, but takes an eclectic approach by drawing on elements from a task-based syllabus, a text-based syllabus and a contentbased syllabus for the design of the programme and types of syllabi which can be considered as more established and traditional ones. However, the course is not solely designed with the aim of preparing students for workplace demands purely from an 'accommodationist' stance. It also attempts to address some of the more critical approaches to pedagogy, whereby students are gently encouraged to develop a critical awareness of workplace practices in order that change might be implemented, where possible.

\section{5-236 Fortune, Alan (King's College London, UK), Learners' use of metalanguage in collaborative form-focused L2 output tasks.} Language Awareness (Clevedon, UK) 14.1 (2005), 21-39.

This paper looks at the employment of metalanguage by advanced English L2 users engaged in Dictogloss, a form-focused collaborative writing task, and compares it with that in an earlier study of intermediate learners. First, three commonly employed types of metalinguistic term are recognised, listed and compared with those reported in a study of students engaged in meaningfocused interactions. Second, grammatical and lexical Language Related Episodes (LREs) which contain metalanguage are categorised, and their frequency of occurrence is compared. The advanced learners are shown to use metalanguage much more often than their intermediate counterparts, particularly in lexical episodes. Third, the role of metalanguage in enabling learners to attend to form is investigated. The 
examination of transcripts of learner interactions provides evidence that metalanguage can play a facilitative role in focusing attention and deciding which form to use. Learners focus on some language points just once during a Dictogloss task (continuous LREs) but some of their other decisions on choice of form are reviewed on at least one occasion (discontinuous LREs). Advanced learners are shown to be more likely to re-engage with a form when using metalanguage than when not doing so.

05-237 Garner, Mark \& Erik Borg (Northumbria U, UK; mark.garner@unn.ac.uk), An ecological perspective on content-based instruction.

Journal of English for Academic Purposes

(Amsterdam, the Netherlands) 4.2 (2005), 119-134.

Content-based instruction (CBI) has been proposed as an effective method of preparing students for study in English-medium universities. Although CBI has won adherents for its pedagogical effectiveness, a theoretical basis for its success has not been fully articulated. There are a variety of frameworks that may be used to provide this theoretical basis. This paper argues that an ecological framework on language learning and use can provide this theoretical underpinning. A content-based English pre-sessional course illustrates the proposed ecological framework. The course, using a textbook for native-English-speaking undergraduates, helps students learn the expectations of university study through extended focussed reading, discussion and writing. In this way, EAP (English for Academic Purposes) skills are contextualised in an integrated learning process.

\section{5-238 Gourlay, Lesley (Napier U, UK;} I.gourlay@napier.ac.uk), Directions and indirect action: learner adaptation of a classroom task. ELT Journal (Oxford, UK) 59.3 (2005), 209-216.

The extent to which learners conform to the structure, aims and linguistic demands of a task is often seen as the responsibility of the materials writer and/or teacher. Given a logical rubric, well-designed task and clear classroom instructions, it is often assumed that the task will be approached as intended. When a task is enacted differently, learner misunderstanding or confusion is often assumed. However, this conception of the relationship between task and enactment does not take into account the separate contribution learners make in bringing a task to life. This article reports on a study looking at examples of learner task adaptation. It concludes by arguing that a task cannot be considered as a static entity, but can only be evaluated by taking account of active learner decision-making, and the nature of classroom process.

05-239 Granville, Stella \& Laura Dison (U of the Witwatersrand, Johannesburg, South Africa; granvils@iweb.co.za), Thinking about thinking: integrating self-reflection into an academic literacy course. Journal of English for Academic
Purposes (Amsterdam, the Netherlands) 4.2 (2005), 99-118.

This paper describes the work done with first-year students doing a course in English for Academic Purposes at the University of the Witwatersrand in Johannesburg. It is concerned with encouraging students to develop meta-cognitive reflective skills as a means to enhancing learning and developing higherorder thinking. This work emphasises the value of promoting reflection in relation to particular and situated learning tasks. By using their own voices in their reflections, students remain grounded in their existing identities and thus more easily make the transition from their everyday language use to the academic languages required at the University. The research also suggests that reflexivity is a developable capacity - the ability to self-reflect is not separate from the process of coming to know and understand.

\section{5-240 Greidanus, Tine, Bianca Beks (Vrije} Universiteit Amsterdam, the Netherlands; t.greidanus@worldonline.nl) \& Richard Wakely, Testing the development of French word knowledge by advanced Dutch- and English-speaking learners and native speakers.

The Modern Language Journal (Malden, MA, USA) 89.2 (2005), 221-233.

This study examines the quality of word knowledge among advanced learners of French as a second language (L2) by means of a word associates test. It describes the development of word knowledge among six groups of university-level participants, who were (a) native speakers of French and (b) learners of French as a foreign language with two different first languages (L1s), Dutch and English. The format of the test incorporated several new features: the tested words were less frequently used French words; the participants were native speakers of French in addition to two categories of advanced learners of French; the number of associate words (fixed or not fixed) was an independent variable. The findings showed that both native and non-native speakers of French progressed in deep-word knowledge when the results of third- and fourth-year students were compared with those of first-year students. Although the test contained a considerable number of French-English cognates, the L1 English learners did not perform better than the Dutch learners. The words tested were not noticeably more difficult when chosen from the 10,000word level rather than from the 5,000-word level.

\section{5-241 Gumock Jeon-Ellis, Robert Debski \&} Gillian Wigglesworth (U of Melbourne, Australia), Oral interaction around computers in the project oriented CALL classroom. Language Learning \& Technology (U of Hawaii, Manoa, USA) 9.3 (2005), 121-145.

Language teachers need to provide students with a context for genuine communication (Sullivan 2000). 
Project-oriented computer-assisted language learning (PrOCALL) attempts to achieve this by orienting learners towards tasks, which encourages them to communicate in the target language while working towards completion of a project (Debski 2000). The study investigates the oral interaction that takes place in this context. According to Vygotsky, social interaction mediates cognitive development. Swain's (2000) application of this concept to language learning suggests that collaborative dialogues mirror the moments of language development. Using this framework, the present study identifies 'language related episodes' (Swain \& Lapkin 1998) and describes the characteristics of the oral interaction generated by two small groups of French learners working towards the completion of Web pages in a major Australian university. The study also describes instances of 'triadic interaction' (van Lier 2002) involving learners' interactions with each other and with the computer screen. In sum, the analysis suggests that the PrOCALL context can provide students with opportunities for collaborative dialogues, through which language learning occurs. However, the social context of these interactions is mediated by personal relationships, preferences, and motivations.

\section{5-242 Haig, Yvonne, Oliver Rhonda \& Judith Rochecouste (Edith Cowan U, Australia), Adolescent speech networks and communicative competence. English in Australia (Norwood, Australia) 141 (2004), 49-57.}

Writing competence has been deemed more significant than oral competence for adolescents in Australian schools. Many teachers are not confident in providing learning opportunities and assessing students' competence in oral language and the assessment tends to be measured through class presentations and judged against written norms. Furthermore there is a mismatch between home and school discourse and a need for students to realise that control of society's dominant discourses allow access to power in society. This study investigates 97 adolescents' communicative competence by asking students to observe their own speech habits and report these to the researchers. Participants were shown web-based material on how to record dense, loose and multiplex networks of relationships and used this knowledge to examine their own networks of family, friends, fellow students and other people they met on a regular basis. The adolescents recognised that ties can change with time and circumstance, though this was not described in the background material. The communicative competence and metalinguistic awareness demonstrated by the participants could be extended in learning programs to help them understand particular uses of language and investigate the way language is used in their community and in other institutions.

05-243 Harwood, Nigel (U of Essex, UK; nharwood@essex.ac.uk), What do we want EAP teaching materials for? Journal of English for
Academic Purposes (Amsterdam, the Netherlands) 4.2 (2005), 149-161.

This paper explores the various anti-textbook arguments in the literature to determine their relevance to the field of English for Academic Purposes (EAP). STRONG and WEAK anti-textbook lines are identified and then a review is made of the corpus-based studies which compare the language EAP textbooks teach with corpora of the language academic writers use. After problematising the methodology of some of the studies, it is claimed that the weak anti-textbook line most accurately describes the state of the EAP textbook market. Although this view is not against textbooks per se, it holds that the current state of commercial materials is highly unsatisfactory, and that publishers and materials writers must make greater efforts to ensure that research findings are operationalised in textbooks. Finally, an EAP textbook is identified which incorporates research findings into its materials and avoids the dangerous generalisations about academic writing which are found in many of its rivals.

\section{5-244 Heift, Trude (Simon Fraser U, Canada; heift@sfu.ca.), Inspectable learner reports for web-based language learning. $R e C A L L$ (Cambridge, UK) 17.1 (2005), 32-46.}

This paper presents a report of a Web-based tutoring system for German - an interface to a persistent learner report that students can inspect and manipulate. The learner report collects and retains information on the learner's progress and performance that is saved between visits. It also provides access to a journaling system that records prior inputs along with detailed error analysis, and a bookmarking system that tracks exercise completion. Finally, results can be printed or emailed to an instructor. In the Fall semester of 2003, a study was conducted with 87 learners of German to investigate how their study habits are affected by an inspectable learner report. The study indicates that students reviewed their learner profiles frequently 33 times on average over the course of a semester and that $70 \%$ of the students repeated exercises after having inspected their learner profile. While detailed user profiles and performance tracking are invaluable tools for CALL (computer-assisted language learning) researchers, this study suggests that students also utilise and are influenced by the information.

\section{5-245 Ibrahim, Nizar (Lebanese U, Lebanon; pronizar@yahoo.com) \& Susan Penfield, Dynamic diversity: new dimensions in mixed composition classes. ELT Journal (Oxford, UK) $\mathbf{5 9 . 3}$ (2005), 217-225.}

This study investigates the effect of a mixed composition class in an American university on student interaction and writing over a semester. The class was a freshman academic writing course that consisted of fifteen international and ten American students. Some teachers believe that a mixed composition class enhances cultural 
understanding, and that cultural diversity presents a good learning environment, contributing to developing student writing and language skills; others believe the contrary because the needs of the English-as-asecond-language (ESL) speakers and native English speakers (NES) cannot be addressed simultaneously. To investigate this issue, we used student journals, interviewed students and observed the class. This paper describes the change in students' attitudes and interaction, the benefits that students gained from the course, and the teachers'/researchers' approach to teaching the course.

\section{5-246 Jepson, Kevin (Monterey Institute of International Studies, USA), Conversations - and negotiated interaction - in text and voice chat rooms. Language Learning \& Technology (U of Hawaii, Manoa, USA) 9.3 (2005), 79-98.}

Despite the expanded use of the Internet for language learning and practice, little attention if any has been given to the quality of interaction among English L2 speakers in conversational text or voice chatrooms. This study explored the patterns of repair moves in synchronous non-native speaker (NNS) text chat rooms in comparison to voice chat rooms on the Internet. The following questions were posed: (a) Which types of repair moves occur in text and voice chats; and (b) what are the differences, if any, between the repair moves in text chats and voice chats when time is held constant? Repair moves made by anonymous NNSs in 10 five-minute, synchronous chat room sessions (5 textchat sessions, 5 voice-chat sessions) were counted and analysed using chi-square with alpha set at .05. Significant differences were found between the higher number of total repair moves made in voice chats and the smaller number in text chats. Qualitative data analysis showed that repair work in voice chats was often pronunciation-related. The study includes discussion that may affect teachers' and learners' considerations of the value of NNS chat room interaction for second language development.

05-247 Juffs, Alan (U of Pittsburgh, USA; juffs@pitt.edu), The influence of first language on the processing of wh-movement in English as a second language. Second Language Research (London, UK) 21.2 (2005), 121-151.

Adult learners of English as a Second Language who speak Chinese, Japanese or Spanish as a first language (L1), and a comparison group of English native speakers read sentences that contain: (a) ungrammatical wh-extractions that violate island constraints; and (b) grammatical long-distance Subject and Object extractions from finite and non-finite clauses. Wordby-word reading times for each sentence were collected using the self-paced reading technique. Results suggest that the presence or absence of $w h$-movement in the L1 and the headedness of the verb phrase in the L1 cannot be taken to account for all of the variation between the non-native-speaker groups. Severe garden path effects were observed in Subject extractions from finite clauses, but not in extractions from non-finite clauses, suggesting that two finite verbs next to one another may be an important factor in causing parsing breakdown. Individual variation in reading time was not predictable from measures of reading span or word span in either the first or second language.

\section{5-248 Knight, Paul (The Open U, UK;}

\section{P. T. Knight@open.ac.uk), Learner interaction} using email: the effects of task modification. ReCALL (Cambridge, UK), 17.1 (2005), 101-121.

This paper outlines the findings of a research project studying the effects of task modification on learner interaction when using email. Taking as its starting point interactionalist theories of SLA (second language acquisition), it argues that if those interactional features characteristic of negotiation of meaning which have been identified as promoting SLA are to be preserved when tasks are transferred from a face-to-face spoken environment to a computer-mediated written asynchronous environment, then modifications to the tasks need to be made.

\section{5-249 Kondo, Takako ( $U$ of Essex, UK), Overpassivization in second language acquisition. International Review of Applied Linguistics in Language Teaching (IRAL) (Berlin, Germany) 43.2. (2005), 129-161.}

An important problem for a language learner is identifying how properties of argument structure are realised morphosyntactically in the particular language they are learning. Speakers of some L1s overgeneralise the morphosyntactic reflexes of the movement of Theme objects in English to unaccusative intransitive verbs, using passive morphology in such cases. In contrast, it appears that the same L2 learners are less likely to do this with unergative verbs. The present paper addresses the question of whether L2 learners of English with typologically different L1s such as Japanese and Spanish will overgeneralise passive morphology to unaccusative verbs in the same way. The results show that both Japanese and Spanish learners of English differentiate unaccusatives from unergatives. However, the two groups differ in their treatment of unaccusatives with transitive alternants and intransitive alternants, suggesting influence of the morphological properties of the L1.

05-250 Lewin, Beverly A. (Tel Aviv U, Israel; lewinb@post.tau.ac.il), Hedging: an exploratory study of authors and readers identification of 'toning down' in scientific texts. Journal of English for Academic Purposes (Amsterdam, the Netherlands) 4.2 (2005), 163-178.

In preparing students for their future discourse communities, the English for Academic Purposes (EAP) and English for Special Purposes (ESP) literature has shown interest in the role of hedges in scientific 
literature. This interest has resulted in several studies that define and classify hedges, and hypothesise about their purpose. Using this work as a theoretical basis, this paper focuses on the possible relation to actual practice. In Study I, authors of scientific texts were asked to identify hedges in their own texts and their motivation for using them. In Study II, three of these texts were presented to EAP students (Ph.D. candidates), requesting them to identify the hedges. There are three main findings. Firstly, the authors' choices of hedges did not coincide with definitions previously reported in the literature. Secondly, the authors did not cite politeness as a general motivation for hedging. Thirdly, there was a great divergence between readers' and authors' identifications. In general, readers identified many more instances of hedges than were intended by the authors. If validated on a larger scale, these findings have implications for instruction in reading and writing of scientific articles.

\section{5-251 Malmqvist, Anita (Umeå U, Sweden), How does group discussion in reconstruction tasks affect written language output. Language} Awareness (Clevedon, UK) 14.2/3 (2005), 128-142.

This article reports on a small-scale study involving adult L1 Swedish learners of L3 German. The main aim of the study was to investigate the effects of group interaction on written German output employing the dictogloss technique. Three short texts were selected for reconstruction, the first and third ones individually, and the second one collectively. The group interactions during the reconstruction of the second text were audiotaped. The first stage of the analysis compared the individually reconstructed with the collectively reconstructed ones, and differences in length as well as in complexity between the two categories were demonstrated. In the second stage the audiotaped interactions were analysed with respect to what features were focused on in the identified language-related episodes (LREs), content/meaning, lexical or grammar. The results show that the majority of the LREs were directed towards grammar, more specifically towards the noun phrase, with case and gender as the most frequently negotiated issues. The last stage of the analysis concerned the outcomes of the LREs, relating them to the participants' proficiency levels and personality traits, as well as to interpersonal dynamics.

05-252 Menard-Warwick, Julia (U of California, USA; jemwarwick@ucdavis.edu),

Intergenerational trajectories and sociopolitical context: Latina immigrants in adult ESL. TESOL Quarterly (Alexandria, VA, USA) 39.2, 165-186.

In this ethnographic study, I contrast the educational experiences of two Central American immigrant women in an English as a second language (ESL) family literacy program in the San Francisco Bay area in 2002. Based on life-history interviews and classroom observations, I argue that these learners' second language and literacy development can only be under- stood within the larger sociopolitical context over time. To this end, I draw on participants' life-history narratives to situate their experiences of studying English within the larger social history of immigration in California and within the intergenerational trajectories of education in their families. Specifically, these narratives illustrate participants' perspectives on how their language learning opportunities have been mediated by such factors as their parents' messages about education, their previous experiences of schooling, US immigration policies, the 2001 economic downturn, and the availability of bilingual education for their children. I conclude by arguing that to meet the diverse needs and goals of learners in their classrooms, ESL educators need to incorporate into the curriculum the specific sociocontextual issues that these learners confront in their daily lives.

\section{5-253 Mirzaiean, Vahid \& Alan Ramsay (Tehran, Iran), Content-based support for Persian learners of English. ReCALL (Cambridge, UK) 17.1 (2005), 139-154.}

People learning a new language have problems with various aspects of the language they want to learn: with its grammar, with its pronunciation, with its morphology, etc. CALL (computer-assisted language learning) tools have been provided for most of these linguistic levels, but comparatively little has been done about helping students with problems of meaning. We argue that language technology is now mature enough to support tools that address at least some semantic issues, and we describe a tool that attempts to provide appropriate support for a specific class of learners.

\section{5-254 Morrison, Bruce (The Hong Kong} Polytechnic U, Hong Kong, China), Evaluating learning gain in a self-access language learning centre. Language Teaching Research (London, UK) 9.3 (2005), 267-293.

If it is to be argued that self-access language learning centres (SACs) provide an effective alternative or complement to more traditionally accepted modes of language learning and teaching, it remains a serious concern that there is no research-based framework specifically developed for their evaluation. It is within the context of a study into the development of such a framework that this paper considers the issue of evaluating learning gain in a SAC. Experience and research suggest that the issue of learning gain is crucial to any discussion of SAC evaluation. There is, however, a tension between the perceived need to include learning gain as a focus in an evaluation of a SAC's effectiveness, and recognition of there being practical problems in so doing. After briefly examining relevant literature, this paper will discuss data derived from the aforementioned study. It will present a number of reasons for attempting to evaluate learning gain, before going on to identify potential difficulties involved and discussing possible ways of achieving this. It will finally suggest that, in order to evaluate learning gain 
within a SAC, there is a need to re-conceptualise what is perceived as learning gain and to look to the learner to identify evidence of such.

\section{5-255 Murphy, Linda (The Open U, UK), Attending to form and meaning: the experience of adult distance learners of French, German and Spanish. Language Teaching Research (London, UK) 9.3 (2005), 295-317.}

The relationship between attention to linguistic form and accuracy or to meaning and fluency in language learning has been explored by language learning researchers for many years. They point to the potential difficulties for learners in attending to form and meaning at the same time and the importance of exposure to meaningful target language communication outside the classroom for 'noticing' forms made salient during instruction. These issues have rarely been considered in a distance-learning context. This paper examines whether part-time distance learners, studying with the UK Open University, engage in such meaningful communication beyond their course of study and how they do this, whether they focus on form or meaning during this activity and the impact it has on their studies. It also explores whether students attend equally to form and meaning when completing assignments as intended by the course teams. The paper concludes by considering the implications of the findings from this investigation for distance language course and assessment design.

\section{5-256 Oliver, Rhonda, Yvonne Haig (Edith} Cowan U, Australia; rhonda.oliver@ecu.edu.au) \& Judith Rochecouste, Communicative competence in oral language assessment. Language and Education (Clevedon, UK) 19.3 (2005), 212-222.

This paper reports on a review of the teaching and assessment of oral language in Western Australian secondary schools. Results show that teachers have considerable difficulty in incorporating oral language tasks into their pedagogy because of a curriculum biased towards developing writing skills. Teachers also revealed that they do not have the skills to assess oral language even though they acknowledge the importance of their students' communicative competence both within the school environment and outside it. Students involved in the study were also aware of many weaknesses in their communicative competence and that these were not being addressed in the classroom.

05-257 Papadopoulou, Despina (Aristotle $U$ of Thessaloniki, Greece), Reading-time studies of second language ambiguity resolution. Second Language Research (London, UK) 21.2 (2005), 98-120.

This article provides a review of studies that have examined the ambiguity resolution strategies employed when processing a second language (L2). The way second language learners parse the L2 input has not yet been thoroughly investigated, although recently there has been an increasing interest in this area. The exploration of the mechanisms L2 learners use to parse ambiguous constructions allows us to examine not only aspects of L2 acquisition that still remain obscure, but also the validity of existing theories of parsing. The studies reported in this article look at three different types of ambiguous construction in the L2, and their results are discussed in relation to the L2 performance pattern. Most of the findings show that even advanced L2 learners are slower readers than native speakers and apply processing routines that depart from those best suited for processing the target language input. In addition, although L2 learners show sensitivity to lexical cues such as verb-argument structure when processing the L2 input, they are less likely to rapidly employ structural information on line.

\section{5-258 Payne, Scott J. \& Brenda M. Ross} (Pennsylvania State U, USA), Synchronous CMC, working memory, and L2 oral proficiency development. Language Learning \& Technology (U of Hawaii, Manoa, USA) 9.3 (2005), 35-54.

A number of recent quasi-experimental studies have investigated the potential of a cross-modality transfer of second language (L2) competency between realtime, conversational exchange via text and speech. Payne \& Whitney employed Levelt's (1989) model of language production and concepts from working memory as a rationale for a hypothesised connection between synchronous computer-mediated communication (SCMC) and second language speech and as a basis for predicting the differential contributions of SCMC to the L2 oral proficiency development. This study extends the psycholinguistic framework reported in Payne \& Whitney (2002) with discourse and corpus analytic techniques to explore how individual differences in working memory capacity may affect the frequency of repetition and other patterns of language use in chatroom discourse. Working memory capacity was measured by a reading span and nonword repetition test. Oral proficiency was measured with a speaking task that solicited a five-minute speech sample and was scored based on a holistic scale. The data collected from twenty chat sessions were analysed for occurrences of repetition and relexicalisation, as well as language output measures. Findings suggest a connection between working memory and language output as measured in this study.

\section{5-259 Rogerson-Revell, Pamela $(U$ of} Leicester, UK; pmrr1@le.ac.uk), A hybrid approach to developing CALL materials: authoring with Macromedia's Dreamweaver/Coursebuilder. ReCALL (Cambridge, UK) 17.1 (2005), 122-138.

This paper describes some of the pedagogical and technical issues involved in adopting a hybrid approach 
to CALL (computer-assisted language learning) materials development. It illustrates some of these issues with reference to a vocational CALL project, LANCAM, which took such a hybrid approach. It describes some of the benefits and considerations involved in hybrid development and evaluates the commercial web development tool, 'Dreamweaver', and its extension application, 'Coursebuilder', which was used to author the LANCAM materials. It argues for a reconsideration of generic web development tools in CALL authoring and also for language experts to be actively involved in the instructional design process.

\section{5-260 Smith, Ross (PricewaterhouseCoopers, Spain), Global English: gift or curse? English Today (Cambridge, UK) 21.2 (2005), 56-62.}

The status of the English language as the lingua franca of the global information society is beyond dispute, but what is not so clear is whether it is capable of fulfilling that role efficiently. In this paper, the author responds to the supporters of Global English by examining the qualities that an ideal international auxiliary language would possess and then discussing the extent to which those qualities are to be found in modern English. After questioning the ease with which English is learned as a lingua franca, it is concluded that the English language is hard to pronounce, irregular and over-complex, making it difficult to learn. In addition, it is said to resemble no other language closely enough for there to be a group of 'natural learners' among other language speakers, as occurs, for instance, among Romance or Nordic languages.

\section{5-261 St-Hilaire, Aonghas (Washington, DC, USA), Louisiana French immersion education: cultural identity and grassroots community development. Journal of Multilingual and Multicultural Development (Clevedon, UK) $\mathbf{2 6 . 2}$ (2005), 158-172.}

This paper examines the role that cultural identity and grassroots activism and community development have played in the creation, maintenance and expansion of Louisiana French immersion education. Although linguists tend to portray the extinction of endangered minority languages as inevitable, research on the effects of minority language immersion education provides evidence that this portrayal is not an accurate one. In Louisiana, community concerns over the imminent death of Louisiana French and its associated culture have mobilised parents, educators and other advocates to establish French immersion programmes in public schools throughout southern Louisiana. Issues of regional and familial cultural identity have provided much of the impetus to expand these programmes in the region. As a result of the successful communitybased efforts to create and extend immersion education in Louisiana, there is now a growing youth population proficient in Louisiana French, providing hope that the region's unique Francophone culture remains an enduring, active presence.
05-262 Todd, Richard W. (King Mogkut's U of Technology, Thailand; irictodd@kmutt.ac.th), 'In an aeroplane, yes, in an aeroplane': within-unit repetitions in classroom discourse. RELC Journal (Thousand Oaks, CA, USA) 36.2 (2005), 189-209.

This paper looks at the functions of repetition of lexical items which fall within a single T-unit. Examining data from a foundation English course at a Thai university, transcripts of eliciting transactions from 12 lessons were divided into T-units. Within-unit repetitions were identified and categorised. Categories of repetition found, in order of frequency, were emphasis, clarification, affirmation, left-fronting, choral units, re-initiation, and pedagogic reformulation. These functions were found to be related to the discourse move the repetition occurs in and to the type of repetition, but were not related to individual teachers. The findings suggest that within-unit repetition is primarily used to aid students' comprehension of teacher talk.

\section{5-263 Uschi, Felix (Monash U, Australia;} uschi.felix@arts.monash.edu.au), E-learning pedagogy in the third millennium: the need for combining social and cognitive constructivist approaches. ReCALL (Cambridge, UK) 17.1 (2005), 85-100.

An analysis of the characteristics of education and educational institutions of the third millennium shows that predominant features are flexibility, inclusiveness, collaboration, authenticity, relevance and extended institutional boundaries. Roles of both students and teachers have changed significantly as educational goals have broadened to include lifelong learning, global interaction, the acquisition of meta-cognitive knowledge and skills, and processes include negotiated curricula and real-life tutors and informants. This is a demanding package that appears to lead us naturally to a social constructivist paradigm for learning and teaching. While few would dispute the value of this approach in humanistic terms, a series of dilemmas social, conceptual, political, pedagogical - have been articulated. This article demonstrates that although these are not insurmountable, addressing them has major time implications. The paper argues that to free up time we need to combine social constructivist activities with cognitive constructivist ones, incorporating personalised ICALL systems.

\section{5-264 Volle, Lisa M. (Central Texas College, USA), Analyzing oral skills in voice and e-mail and online interviews. Language Learning \&} Technology (U of Hawaii, Manoa, USA) 9.3 (2005), 145-163.

This study investigated the acquisition of speaking skills in an online distance education course of nineteen first-semester Spanish learners. The possibility of oral development in a strictly online course was examined based on students' pronunciation production in two 
types of recorded speaking activities and in two realtime conversations. Students created two types of voiced audio emails each week during the semester: read aloud passages and grammar-drill completions. In order to determine whether students' pronunciation really improved over the course of the semester, their performance on these two types of audio emails at the beginning of the semester was compared with that at the end of the semester. In addition, students participated in two Internet-mediated oral conversations with their instructor using MSN messaging at the mid-term week and final week of the semester. Three kinds of data were collected from the conversations: an articulation score (articulation $=$ pronunciation, stress and intonation), an accuracy score and a proficiency score. Students' performance on these interviews provided further evidence regarding the development of their oral skills. The findings suggest that only in the area of oral proficiency were there significant gains in scores.

\section{5-265 Williams, John N. (Cambridge U, UK; jnw12@cam.ac.uk), Learning without awareness. Studies in Second Language Acquisition (Cambridge, UK) 27.2 (2005), 269-304.}

Two experiments examined the learning of formmeaning connections under conditions where the relevant forms were noticed but the critical aspects of meaning were not. Miniature noun class systems were employed and the participants were told that the choice of determiner in noun phrases depended on whether the object was 'near' or 'far' from the subject of the sentence. What they were not told was that the choice of determiner also depended on the animacy of the noun. Most participants remained unaware of this correlation during the training and test tasks; yet when faced with a choice between two determiners for a noun, they chose the one that was appropriate to the noun's animacy at significantly above-chance levels, even though that combination had never been encountered during training. This ability to generalise provided evidence of learning form-meaning connections without awareness. In both experiments, there was a correlation between generalisation test performance and knowledge of languages that encode grammatical gender. This points to the importance of prior knowledge in implicit learning.

\section{5-266 Yongqi Gu, Peter, Guangwei Hu \& Lawrence Jun Zhang (Nanyang Technological U, Singapore; yqpgu@nie.edu.sg), Investigating language learner strategies among lower primary school pupils in Singapore. Language and Education (Clevedon, UK) 19.4 (2005), 281-303.}

To date, very few empirical studies can be found on primary, especially lower primary school pupils' use of language learner strategies. The few studies that exist often employ research techniques such as questionnaires and think-aloud protocols as studies on adults do.
However, it is not yet clearly understood whether the research methods commonly used with adult learners can be applied to lower primary school pupils (grades 1-3). Practically no published study in this area reports the problems associated with eliciting language learner strategy data from children. In addition, not much is known about whether children as young as primary one can be differentiated in terms of learner strategies they use in completing language tasks. This paper shares the problems encountered in eliciting strategies from lower primary school pupils in Singapore and presents some preliminary findings on these learners' use of language learner strategies.

\section{Reading and writing}

doi:10.1017/S0261444805232998

05-267 Aitchison, Claire (U of Western Sydney, Australia), Thesis writing circles. Hong Kong Journal of Applied Linguistics (Hong Kong, China) 8.2 (2003), 97-115.

Numerous studies show that postgraduate research students report high levels of frustration with the traditional western approach to doctoral writing that involves a singular focus on an extended piece of writing undertaken in isolation and frequently monitored by only one or two supervisors. For students with a non-English-speaking background these frustrations are often magnified by linguistic and cultural differences. This paper reports on a new writing program, the 'Thesis Writing Circle', which attempts to provide a new type of support for doctoral students. The Circle draws on the notion of peer support and review by guiding participants to share their writing on a weekly basis. Each session includes also a teacherled component whereby specific skills of high-level academic writing are scaffolded and discussed. The role of the facilitator is crucial in this small group approach, as students must actively and sensitively participate as developing writers as well as learn how to critically appraise the work of others. By engaging in guided textual analysis, involving a close up review of whole and partial texts, students learn in a very personal way about their strengths and weakness as both writers and critical reviewers of academic texts.

05-268 Allison, Desmond (The National $U$ of Singapore), Authority and accommodation in higher degree research proposals. Hong Kong Journal of Applied Linguistics (Hong Kong, China) 8.2 (2003), 155-180.

This paper explores the textual production of an important but elusive written genre, the higher degree research proposal, by writers of English as a Second Language (ESL) seeking admission to graduate programmes in an English-medium university in SouthEast Asia. The account takes the difficult notions of authority and accommodation as its dialectical points of reference. Effective communication in a higher 
degree research proposal calls for an assured display of knowledge, yet most proposal writers lack the experience of disciplinary cultures, as well as the institutional power, of the readers they seek to convince. The rhetorical challenge of research proposal writing is therefore both acute and distinctive. The data for this study comprise four successful and four unsuccessful research proposal texts that writers have made available for research. Focusing primarily upon aspects of lexical choice and text organisation, the discourse analysis evokes tensions at work as writers seek both to accommodate stated expectations and to establish a sense of authority within the context of the discipline. A closing discussion brings together some pragmatic and critical implications for the teaching and practice of research communication within English for Academic Purposes (EAP).

\section{5-269 Bazerman, Charles (U of California, Santa Barbara, USA), An essay on pedagogy by Mikhail M. Bakhtin. Written Communication} (Thousand Oaks, CA, USA) 22.3 (2005), 333-338.

This is an extended summary of a pedagogic essay by Mikhail M. Bakhtin on writing style, entitled 'Dialogic origin and dialogic pedagogy of grammar: stylistics as part of Russian language instruction in secondary school'. In this essay, written in spring 1945, while Bakhtin was a secondary school teacher of Russian language arts, he argues that every grammatical form is a representation of reality and needs to be taught in relation to stylistic choices; otherwise, grammar instruction is pedantic and leads students to write in a deadening bookish style. Bakhtin describes and analyses a lesson on the stylistic force of parataxic sentences. He asks students to identify the voice and psychological expression conveyed in examples from Pushkin and Gogol, so they may recover the liveliness in their expression that they had in their younger grades, but at a higher level of cultural development. He finds that after instruction, students use more parataxic sentences, increasing the liveliness of their writing.

\section{5-270 Belanger, Joe (U of British Columbia, USA), 'When will we ever learn?': the case for formative assessment supporting writing development. English in Australia (Norwood, Australia) 141 (2004), 41-48.}

In Australian secondary and post-secondary education, tutors usually give summative assessment of writing and have the dual role of suggesting improvements while giving a final judgement. This paper uses a recent Canadian study reporting the think-aloud responses of 53 secondary school students as they read their teachers' comments (J. Belanger \& P. Allingham 2003, 'A technical report: using think-aloud methods to investigate the processes secondary school students use to respond to their teachers' comments on their written work'). In this study students were more concerned with the grade given than with teachers' comments. Students who received the grade they expected often did not read the comments; those who did read the comments saw them as a rationale for deducting marks rather than a means of improving their writing. There was little understanding of error codes and students did not like stylistic revisions of their sentences. Frequently, marked papers were never looked at again, with students seeing each writing assignment as a separate task. However comments on assignments early in the term tended to be read and used to work out what the teacher valued and are thus the best opportunity for effecting change. The authors suggest that if the roles of formative and summative evaluation are separated then teachers can better engage in dialogue with students about their work.

\section{5-271 Bodwell, Mary Buchinger}

(Massachusetts College of Pharmacy and Health Sciences, USA; mary.bodwell@bos.mcphs.edu),

'Now what does that mean, "first draft"?': responding to text in an adult literacy class. Linguistics and Education (Amsterdam, the Netherlands) 15.1-2 (2004), 59-79.

This paper examines a discussion among a teacher and four Latin-American women in a family literacy class who engage for the first time in the practice of peerediting a learner's text. In the analysis, it becomes evident that they have different ideas of and responses to the text. Whereas the teacher's focus is on revising the textual elements, the learners use the text to explore and negotiate their maternal practices. The conclusions are two-fold: pedagogy can benefit from a broadened definition of literate activity; and secondly, classroom practices, such as peer-editing, are social constructs and need to be explicitly scaffolded.

\section{5-272 Broadley, Guy, Seeing forward looking back: the New Zealand literacy picture.}

Australian Journal of Language and Literacy

(Norwood, Australia) 28.1 (2005), 8-18.

This paper begins with a brief overview of reading education in New Zealand from the 1960s onwards. The author is a school psychologist and supervisor of student teachers and gives a personal account of classroom reading lessons he has observed. He suggests that children in New Zealand should be provided with a range of strategies for reading and notes how this country's recent poorer international performance in reading compares to that of other countries. Among those who have shown the least improvement are Maori and Pacifica children who are frequently among the poorer children in a school. Broadley suggests that the quality of teachers' knowledge of current literacy practices is highly important and points to the need for more in-service training courses, pointing out that many of these can now be completed online.

\section{5-273 Bruton, Anthony \& Emilia Alonso} Marks (Universidad de Sevilla, Spain), Reading 
texts in instructed $L 1$ and FL reading: student perceptions and actual selections. Hispania (Exton, PA, USA) 87.4 (2004), 770-783.

Extensive reading is generally agreed to be crucial in improving students' reading and vocabulary in a foreign language, and it is usually assumed that students read narratives. There is, however, a distinct lack of research into students' perceptions of what they read. This paper examines the perceived reading wants and needs of forty 16-18 year old students of Spanish as a foreign language (FL) in the United States and compares the results with those of a study of 55 similar students of English as a FL in Spain (G. Alvarez, A. Broca \& A. Bruton 1993, 'Reading wants and needs in EFL: learners' and teachers' perceptions', Reading in a Foreign Language 9.2, 859-865). Both Spanish and American students were given two questionnaires in their own language (L1) about this language and about their FL. The results indicated that perceived wants outweigh the perceived needs for reading both in the FL and in the L1, with the exception of English as an FL, where the two appeared very similar. The authors discuss the need for teachers to take students' reading needs and wants into consideration so that reading competency is increased and the desire to read is encouraged in both FL and L1.

\section{5-274 Chandrasegaran, Antonia (Nanyang Technical U, Singapore), Mary Ellis \& Gloria Poedjosoedarmo, Essay Assist: developing software for writing skills improvement in partnership with students. RELC Journal} (Thousand Oaks, CA, USA) 36.2 (2005), 137-155.

School- and university-based writing tasks make demands on cognitive processing and socio-cultural knowledge that vary with the discipline in which the writing is situated. The variation in socio-cognitive demands makes the use of self-accessed, computer-mediated writing instruction more promising than conventional, teacher-fronted instruction. To be effective, self-accessed writing instruction should be constructed in partnership with student writers whose composing problems would form the basis of the content and organisation of the instructional package. This paper demonstrates how a socio-cognitive model of writing informs the development of Essay Assist, a computer program for guiding students towards appropriate decisions in the essay writing process. Results of a preliminary trial are reported and their implications discussed.

\section{5-275 Chujo, Kiyomi (Nihon U, Japan;} chujo@cit.nihon-u.ac.jp) \& Masao Utiyama, Understanding the role of text length, sample size and vocabulary size in determining text coverage. Reading in a Foreign Language (Honolulu, HI, USA) 17.1 (2005), 1-22.

Although the use of 'text coverage' to measure the intelligibility of reading materials is increasing in the field of vocabulary teaching and learning, to date there have been few studies which address the methodological variables that can affect reliable text coverage calculations. The objective of this paper is to investigate how differing vocabulary size, text length and sample size might affect the stability of text coverage, and to define relevant parameters. In this study, twenty-three varying vocabulary sizes taken from the high frequency words of the British National Corpus and twenty-six different text lengths taken from the Time Almanac corpus were analysed using ten different sample sizes in 1000 iterations to calculate text coverage, and the results were analysed using the distribution of the mean score and standard deviation. The results of the study empirically demonstrate that text coverage is more stable when the vocabulary size is larger, the text length is longer and more samples are used. It was also found that the stability of text coverage is greater from a larger number of shorter samples than from a fewer number of longer samples. As a practical guideline for educators, a table showing minimum parameters is included for reference in computing text coverage calculations.

\section{5-276 Cromley, Jennifer G. \& Roger Azevedo} (U of Maryland College Park, USA), What do reading tutors do?A naturalistic study of more and less experienced tutors in reading. Discourse Processes (Mahwah, NJ, USA) 40.1 (2005), 83-113.

Virtually every study of tutoring in reading has found it to be highly effective, but the reasons for its effectiveness are relatively unexplored. This study builds on a small body of research on the tutoring process in reading and a large body of research from other well-defined domains by collecting and analysing verbal protocols from three more experienced and three less experienced volunteer tutors from pre-existing dyads as they taught adults with decoding problems. It was found that more experienced tutors used significantly more cognitive scaffolding and significantly less motivational scaffolding than did less experienced tutors. Tutors were similar in their rates of question asking, content errors and responses to student errors. The authors suggest some hypotheses for modelling in the design of a computer-based readingdecoding tutor and for training human tutors who teach decoding.

\section{5-277 Crompton, Peter}

(crompton@fastmail.fm), 'Where', 'In which', and 'In that': a corpus-based approach to error analysis. RELC Journal (Thousand Oaks, CA, USA) 36.2 (2005), 157-176.

This paper analyses the use of the word where in texts written by Malay-speaking learners of English. Data from a learner corpus are compared with data from two corpora of academic writing by native English-speaker writers. Considerable over-use of where is found: this is explained as a consequence of various patterns of misuse of where and non-use of standard forms of relativisation 
common in written academic English. Suggestions are offered as to (a) the causes of misuse and (b) teaching strategies for helping students avoid misuse.

05-278 Day, Richard (U of Hawaii, Manoa, USA) \& Jeong-suk Park, Developing reading comprehension questions. Reading in a Foreign Language (Honolulu, HI, USA) 17.1 (2005), 60-73.

This article presents a detailed picture of six types of comprehension and five forms of questions that can be used to help students become interactive readers. The taxonomies of the types of comprehension and the forms of questions may also be used as a checklist for language teachers as well as materials developers. Teachers can use the taxonomies to make their own comprehension questions for texts that their students read to help them understand better what they read. In addition, they can be used to analyse instructional materials and to develop materials to ensure that the various forms of questions are used to help students respond to a variety of types of comprehension. Appendices contain example questions, allowing focus on their form and on the type of comprehension, and answers based on the article itself, and a text for intermediate learners.

\section{5-279 Dunlosky, John \& Katherine A.}

Rawson (U of North Carolina at Greensboro, USA), Why does rereading improve metacomprehension accuracy? Evaluating the Levels-of-Disruption Hypothesis for the

Rereading Effect. Discourse Processes (Mahwah, NJ, USA) 40.1 (2005), 37-55.

Rereading can improve the accuracy of people's predictions of future test performance for text material. This research investigated this rereading effect by evaluating two predictions from the levels-of-disruption hypothesis: (a) the rereading effect will occur when the criterion test measures comprehension of the text, and (b) the rereading effect will not occur when a one-week delay occurs between initial reading and rereading. Participants $(\mathrm{N}=113)$ were assigned to one of three groups: single reading, immediate rereading and rereading after a one-week delay. Outcomes were consistent with the two predictions stated earlier. This article discusses the status of the levels-of-disruption hypothesis and alternative hypotheses based on the cognitive effort required to process texts.

05-280 Guillot, Marie-Noëlle (U of East Anglia, UK), II y a des gens qui disent que... 'there are people who say that ...' Beyond grammatical accuracy in FL learners' writing: issues of non-nativeness. International Review of Applied Linguistics in Language Teaching (IRAL) (Berlin, Germany) 43.2. (2005), 109-128.

This paper draws on data used in the pilot application of data-driven pedagogical strategies for dealing with non-nativeness in the writing of advanced FL (foreign language) learners of French to expose attendant practical problems and theoretical issues. The discussion focuses on the question of vagueness in academic writing and involves a three-way comparison of FL French data, NS (native speaker) target language data and NS mother tongue data. This contrastive approach entails assessing the relationship between speech and writing in the diagnosis of sources of non-nativeness, and the influence of both interlingual and intralingual transfer. It underscores the interrelatedness of discourse and psycholinguistic factors generally and, more specifically, the likely psycholinguistic relevance to questions of non-nativeness and written phraseology of contrasts in cognitive and discourse constraints between speech and writing; it also points to limitations of classroom data likely to be used in pedagogical applications, and suggests that principled guidance from research is needed if these questions are to be satisfactorily dealt with in teaching.

\section{5-281 Haan, Pieter de (p.dehaan@let.ru.nl) \& Kees van Esch, The development of writing in English and Spanish as foreign languages.}

Assessing Writing (Amsterdam, the Netherlands)

10.2 (2005), 100-116.

This article presents the first results of the study of argumentative essays in English as a foreign language (L1) and in Spanish as L2 and as L1, carried out at Radboud University, Nijmegen, between 2002 and 2008. The aim of the project is to compare teachers' holistic assessments with the results of the quantitative analysis of syntactic and lexical features of the essays and measure the development in writing skill from one year to the next. The first part of the current article discusses general aspects of L1 and L2 writing, and establishes the place of our project in the discipline. The second part presents the methodology adopted and the results of the quantitative analyses that have been carried out so far.

04-282 Hitosugi, Claire lkumi \& Richard R. Day (U of Hawaii, Manoa, USA), Extensive reading in Japanese. Reading in a Foreign Language (Honolulu, HI, USA) 16.1 (2004), 21-39.

This article discusses how the authors incorporated an extensive reading (ER) program into the second semester of a class of fourteen first-year Japanese course at the University of Hawaii using Japanese children's literature. After summarising the ten principles of ER, the authors describe how they addressed six critical issues faced while introducing ER into the course. The outcomes of this ten-week program, which showed that the students improved their scores according to a traditional measure of reading comprehension, are also discussed. Positive results were also found in an affective questionnaire that measured attitudes toward and motivation for learning Japanese.

05-283 Hunt, Alan (Kansai U, Osaka, Japan) \& David Beglar, A framework for developing EFL 
reading vocabulary. Reading in a Foreign

Language (Honolulu, HI, USA) 17.1 (2005), 23-59.

Effective second language vocabulary acquisition is particularly important for English as a foreign language (EFL) learners who frequently acquire impoverished lexicons despite years of formal study. This paper comprehensively reviews and critiques second language (L2) reading vocabulary research and proposes that EFL teachers and administrators adopt a systematic framework in order to speed up lexical development. This framework incorporates two approaches: 1) promoting explicit lexical instruction and learning strategies; and 2) encouraging the use of implicit lexical instruction and learning strategies. The three most crucial explicit lexical instruction and learning strategies are acquiring decontextualised lexis, using dictionaries and inferring from context. Implicit lexical instruction and learning can take many forms, including the use of integrated task sets and narrow reading; however, this framework emphasises extensive reading, which is arguably the primary way that EFL learners can build their reading vocabulary to an advanced level. The principal notion underlying this framework is that the most effective and efficient lexical development will occur in multifaceted curriculums that achieve a pedagogically sound balance between explicit and implicit activities for L2 learners at all levels of their development.

\section{5-284 Jackson, Sue \& Susan Gee (Victoria U of Wellington, New Zealand; sue.jackson@vuw.ac.nz), 'Look Janet', ‘No you look John': constructions of gender in early school reader illustrations across $\mathbf{5 0}$ years.} Gender and Education (Abingdon, UK) 17.2 (2005), 115-128.

Children's school readers have simple storylines and are experienced by all children, making them an important cultural resource through which gender identity is constructed. However, few studies have considered the contribution of these illustrations in school readers to gender construction. This study covers a randomly selected sample of 100 readers used in New Zealand schools over the last half of the 20th century and analyses how gender is illustrated. Among other factors, the paper considers the 'snuggle factor' in which girls are depicted cradling objects close to their bodies whereas boys are shown holding objects at a distance. This is one means by which readers can show traditional femininity and masculinity. Other methods are the stance and gesture of females and males in illustrations, such as sitting with legs crossed (females) versus both feet on the floor (males), bending down to children (mothers) versus kneeling or crouching (fathers). In terms of appearance, girls can choose to wear trousers and thus align themselves with traditional masculinity. The apron as protector of clothing and indicator of domesticity is also explored. The paper concludes that while children may be resistant to attempts to change traditional representations of gender, it is important that illustrators accept responsibility for providing children with multiple ways of being female and male.

\section{5-285 Kaplan, B. Robert (U of Southern} California, USA) \& Richard B. Baldauf, Jr., Editing contributed scholarly articles from a language management perspective. Journal of Second Language Writing (Amsterdam, the Netherlands) 14.1 (2005), 47-62.

Working from a language management perspective, this article examines problems faced by authors using English as a second language in order to contribute articles to scholarly journals. The article considers problems journal editors have to deal with when working with such authors on language issues. It then explains the theory of language management, outlining simple and organised management along with related characterisics such as metalinguistic discussion. The article draws on a half-million word corpus to illustrate various problems of article authorship and raises important issues that teachers of writing in English as a second/foreign language also need to consider. Among such matters is the ethical dilemma of how acceptable it is for teachers and editors to improve a text. The authors, who are also editors of a well known journal, outline how while the editor's role is ideally to bring to the attention language matters for authors to deal with, final editorial responsibility is to the publisher and subscribers. The article concludes that much of what appears to be simple language management on the part of the editor in fact concerns organised language management that goes beyond making merely cosmetic textual alterations.

\section{5-286 Keen, John (Manchester U, UK;} john.keen@man.ac.uk), Sentence-combining and redrafting processes in the writing of secondary school students in the UK. Linguistics and Education (Amsterdam, the Netherlands) 15.1-2 (2004), 81-97.

This article describes an investigation based on a corpus of original and redrafted versions of extracts from reflective narratives by 15-year-old secondary school students in the UK. It builds on the established research on sentence combining with respect to students' writing development. The findings are discussed in relation to the use of coordinating conjunctions, the subordinating conjunctions as and because, and subordination other than explanatory as and because. They suggest that aspects of grammatical development in students' writing are integrally related to propositional meaning, cohesion and rhetorical effects, and in particular that redrafting can enable students to explore forms of expression in their own writing, that coordination and use of explanatory as and because can enable students to explore relationships between clauses in writing, and that a complex process of rank shift of clause types, including subordinate clauses, can enable students to enhance their clause planning and their ability to elaborate. 
05-287 Liu, Lu (Purdue U, USA), Rhetorical education through writing instruction across cultures: a comparative analysis of select online instructional materials on argumentative writing. Journal of Second Language Writing (Amsterdam, the Netherlands) 14.1 (2005), 1-18.

Recent studies on Chinese-English contrastive rhetoric have argued that there is little to contrast and that the traditional qi (beginning), cheng (transition), zhuan (turning), he (synthesis) structure has little influence on contemporary Chinese writing. This comparative analysis of online materials on argumentative writing for American and Mainland Chinese school writers reveals that although the two groups agree on the purpose, tripartite structure and the use of formal logic, they differ in the discussion of some fundamentals for argumentative writing. The article explains how the American group considers anticipating the opposition a must, while the Chinese group demonstrates epistemological and dialogical emphases and highlights the need to use analogies. Also explained is how the importance of analogies and epistemological and dialogical emphases can be traced to ancient Chinese rhetorical theories. The article argues that these findings may help us to understand the assumptions and beliefs that underlie rhetorical conventions or textual features. Further comparative research on Mainland Chinese and American pedagogical materials on argumentative writing is suggested.

05-288 Liu, Yongbing (Nanyang Technological U, Singapore), The construction of pro-science and technology discourse in Chinese language textbooks. Language and Education (Clevedon, UK) 19.4 (2005), 281-303.

This paper examines the pro-science and technology discourse constructed in Chinese language textbooks currently used for primary school students nationwide in China. By applying analytical techniques of critical discourse analysis, the paper critically investigates how the discourse is constructed and what ideological forces are manifested in the textbooks. More specifically, it analyses how textual features, such as lexical choices, grammatical elements and generic structures, are manipulated in the construction of a version of science and technology written for the child reader. Further, the article explores how the discourse positions child readers to read in ways that the discourse is constructed, and concludes that the discourse serves the interest of the government and its cultural elites but not the interests of the child reader.

05-289 McCarthey, Sarah J. \& Georgia Earnest García (U of Illinois at Urbana-Champaign, USA),

English language learners' writing practices and attitudes. Written Communication (Thousand Oaks, CA, USA) 22.2 (2005), 36-75.

This study of English language learners, six Mandarinspeaking and five Spanish-speaking elementary students, revealed that students engaged in a variety of writing practices at home and school. A continuum of attitudes, from positive to negative, characterised students' attitudes towards writing in English and in L1. Students' writing practices and attitudes toward writing were influenced by home backgrounds and classroom contexts. Home background influences included parents' educational backgrounds and income levels, plans for staying in the United States, support for writing at home, and cultural expectations. School and classroom factors included frequency and quality of opportunities for writing and teachers' expectations for writing tasks. Implications of the study include the necessity to provide multiple opportunities for students to write for purposeful audiences in L1 as well as in English.

\section{5-290 McCarthey, Sarah J., Yuey-Hi Guo}

\& Sunday Cummins (U of Illinois, USA), Understanding changes in elementary Mandarin students' L1 and L2 writing. Journal of Second Language Writing (Amsterdam, the Netherlands) 14.2 (2005), 71-104.

This article reports on a study that focused on five elementary Mandarin-speaking students' development as writers over a two-year period in US classrooms. The article explains how Mandarin speakers who came to the US in $2 \mathrm{nd}$, 3rd or 4th grades demonstrated some language loss in their Chinese writing and how differences were found in terms of sentence complexity, character complexity, rhetorical features, and voice in students' writing between Year 1 and Year 2. The article describes how students' native language loss was mitigated by their initial writing competence, support at home, and opportunities to write in Chinese at school. Four of the five students' English writing made dramatic improvements in grammar and punctuation, sentence complexity, rhetorical style and voice over the two years. The fifth student, whose writing did not improve, had few opportunities to write in his new school setting. Findings suggest that the amount and quality of writing opportunities in each language affect students' writing development.

\section{5-291 Mills, Kathy, Deconstructing binary oppositions in literacy discourse and pedagogy.} Australian Journal of Language and Literacy (Norwood, Australia) 28.1 (2005), 67-82.

This paper presents a personal position statement in relation to three dominant polarities within the field of literacy learning in the last half-century. The writer is a tertiary literacy educator with experience in primary schools and her teaching practice reflects a selection of pedagogical principles. The first polarity concerns skills-based ideology versus the whole language debate. Advocates of the former see literacy as a set of technical skills such as sight vocabulary, perceptual skills and word comprehension to be acquired, while those of the latter emphasise the importance of background knowledge. The second binary opposition is of 
print-based literacy as the focus of reading versus multiliteracies, which extends literacy to cover audio, visual, gestural, spatial and cultural elements. The third polarisation is of cultural heritage, which reproduces dominant cultural values of the past, and critical literacy, which pays attention to unequal power relationships. These competing ideologies are examined closely within the paper and the author concludes that this examination is necessary to reframe literacy pedagogy for the present and future.

\section{5-292 Mišak, Aleksandra, Matko Marušić \& Ana Marušić (Zagreb U School of Medicine, Croatia), Manuscript editing as a way of teaching academic writing: experience from a small scientific journal. Journal of Second Language Writing (Amsterdam, the Netherlands) 14.2 (2005), 151-172.}

Medical writing and manuscript preparation are rarely taught in the context of undergraduate, graduate, or continuing medical education. As editors of a 'small' medical scientific journal published in English in a nonnative English-speaking (NNES) country, the authors hold that the knowledge of scientific methodology and specificities of scientific reporting is a necessary precondition for a successful scientific publication. They explain how their experience shows that language professionals and translators whose services NNES authors use should be acquainted with the basic rules of scientific reporting and describe how each of the four layers of a manuscript - the STUDY QUALITY, the NARRATIVE, the SCIENTIFIC REPORTING STYLE, and finally THE LANGUAGE PER SE - can be improved.

05-293 Misson, Ray (U of Melbourne, Australia), What are we creating in creative writing? English in Australia (Norwood, Australia) 141 (2004), 132-140.

English-speaking primary- and secondary-age schoolchildren in Australia study creative writing throughout their school career; yet there has been little theoretical study of this. This paper discusses 'critical capacity' and 'imagination', and maps the terms on to reception and production, respectively. The former refers to developing a critical understanding of how a text constructs the world while imagination determines the kind of world a writer creates in his or her writing. The paper argues that our critical and imaginative powers are closely linked and that we should be aware of the connection between imagination and receptive skills, and critical capacity and productive skills. The writer goes on to discuss school writing in the classroom as a rehearsal for authentic writing elsewhere. Teachers require students to imagine themselves as the type of person who writes a letter to a newspaper or a book review; thus imagination is necessary within functional writing. A writing curriculum ultimately helps students to create a different persona and operate within different discourses and should be given greater prominence within the school curriculum.

\section{5-294 Nelson, Cynthia D. \& Caroline San}

Miguel (U of Technology, Sydney, Australia), Designing doctoral writing workshops that problematise textual practices. Hong Kong Journal of Applied Linguistics (Hong Kong, China) 8.2 (2003), 116-136.

Academic literacy lecturers are increasingly being called upon to contribute to the education of research postgraduates by designing and teaching writing workshops. However, the literature on this topic rarely addresses the needs of students writing in their first language, or writing about practice-based research for scholarly readers. Moreover, such literature rarely presents a detailed discussion of actual tasks used in writing workshops, or explores how writing theories can be made accessible to students who have little familiarity with linguistics. This paper presents and discusses workshop tasks developed for students undertaking a professional doctorate degree in nursing at an Australian university. The focus of the paper is on designing tasks that problematise textual practices, with a particular emphasis on writer identity in academic discourses. The first part of the paper describes the teaching context and outlines the theories and issues that underpin our teaching approach, drawing on literature about professional doctorates, postgraduate research writing, research writing in the discipline of nursing, and pedagogic approaches. The second part of the paper presents several workshop tasks that illustrate how these theories and issues inform our teaching practices.

\section{5-295 Oller, Jr., John W., Liang Chen,} Stephen, D. Oller \& Ning Pan ( $U$ of Louisiana at Lafayette, USA), Empirical predictions from a general theory of signs. Discourse Processes (Mahwah, NJ, USA) 40.2 (2005), 115-144.

General sign theory (GST) deals with how distinct sign systems are grounded, developed with increasing abstractness over time, and differentiated in efficacies in experience and discourse. GST has three components: The theory of true narrative representations (TNR theory) shows that TNRs are unique in being relatively well determined with respect to the real world, well connected to that world and to each other, and generalisable. TNRs provide the essential grounding for the child's first meaningful words. GST also includes a theory of abstraction about how signs of all kinds are vested with content and a theory of systems grammar that incorporates actual bodily referents. GST is compatible with aspects of amodal, indexical and perceptual symbol theories but predicts certain findings they cannot account for. For example, it differentiates percepts of objects (in their spacetime settings) from icons abstracted from those percepts (as in remembering or anticipating the movement of an object) and from fully generalisable concepts (e.g. the 
unfilled-out thought or concept of an as yet unidentified object).

05-296 Paltridge, Brian (U of Sydney, Australia), Teaching thesis and dissertation writing. Hong Kong Journal of Applied Linguistics (Hong Kong, China) 8.2 (2003), 78-96.

A large number of students in US, Canadian, British and Australian universities are enrolled in a postgraduate degree that requires them to write a thesis or dissertation in English. Many students in countries such as Hong Kong are also required to write a thesis or dissertation in English. For many English native- and non-nativespeaker students, this is the first time they have attempted to write a text of this kind. This article describes a course on dissertation writing that was developed for master's students at the University of Sydney, Australia. The course aims to meet the needs of the students by familiarising them with the conventions and expectations of this particular academic genre, as well as to give them strategies for writing their own dissertations.

\section{5-297 Pantaleo, Sylvia, Young children engage with the metafictive in picture books.} Australian Journal of Language and Literacy (Norwood, Australia) 28.1 (2005), 19-37.

'Metafiction' refers to fictional writing which is inwardlooking in some way, for example, by having a narrator address the reader, having stories within stories or containing parodies of other texts. These devices often distance readers from the text and require readers to move beyond traditional expectations to a more active interpretive role. This study explores 19 elementary school children's responses to and interpretations of eight picture books with metafictive devices. During this nine-week-long Australian study, the children listened to the researcher read the eight books and then were asked to say what they were thinking and feeling as the story session progressed. The books required the children to be more involved in the creation of meaning and to understand the text both inferentially and critically. There were more 'gaps' for the reader to complete in order to fully understand the stories. The study shows that the children could understand the sophisticated narrative devices used and also enjoyed the experience.

\section{5-298 Pearson, Lynn (Bowling Green State U, USA), The web portfolio: a project to teach} Spanish reading and Hispanic cultures. Hispania (Exton, PA, USA) 87.4 (2004), 759-769.

This paper describes a project using web pages on cultural topics to encourage English-speaking university students of Spanish to read both independently and extensively. The Web Portfolio guides students in creating a simple web page on a topic of their choosing with links to other relevant pages. The electronic medium of the portfolio allows the pages to be easily created and revised and the resulting material is accessible to both other learners and the instructor. The project helps to address four possible problems in understanding internet content: lack of background knowledge, lack of knowledge of cultural allusions, the necessity for critical reading skills to determine the reliability of web content and the importance of adopting an appropriate reading strategy. Learners are allowed to choose cultural topics of interest to them and are given help in using the Internet in a foreign language. In creating their own Web Portfolio learners become researchers and use Spanish to gather information which can then be accessed by other students.

\section{5-299 Peterson, Shelley \& Theresa Calovini}

(Toronto U, Canada; slpeterson@oise.utoronto.ca), Social ideologies in grade eight students' conversation and narrative writing. Linguistics and Education (Amsterdam, the Netherlands) 15.1-2 (2004), 121-139.

This study uses systemic functional linguistics and the related critical discourse analysis to examine intertextual links between four eighth-grade students' talk in informal conversations as they generated ideas for the characters of their narrative writing. Data sources included classroom observations, students' drafts and polished writing, student and teacher interviews, and the informal conversations among the two girls and two boys as they generated character descriptions. An analysis of the interpersonal relationships showed that all the students participated in advancing topics for discussion and supported each other relatively evenly. In terms of the ideological content of the talk, it was found that students negotiate gender ideologies along with ideologies of race, ethnicity, socioeconomic status and age. The use of critical discourse analysis opened up space for identifying gender fairness and equity in classrooms through complicating gender relationships within students' interactions around writing.

\section{5-300 Reynolds, Dudley W. (U of Houston, USA), Linguistic correlates of second language literacy development: evidence from middle-grade learner essays. Journal of Second} Language Writing (Amsterdam, the Netherlands) 14.1 (2005), 19-45.

This article compares the development of linguistic fluency in the writing of 5th-8th grade US students enrolled on a course in English as a Second Language (ESL) and regular language arts (RLA) classes. The article defines linguistic fluency as the use of linguistic structures appropriate to rhetorical and social purposes and is measured using five sets of features shown by Reppen (2001 \& 1994) which deal with variation in elementary student language. It explains how the same variational patterns found by Reppen in her corpus are shown to exist in the writing of the ESL and RLA students and demonstrates how more careful 
analysis of the individual features associated with each set indicates that the RLA students hold stronger associations between the features and the rhetorical and social functions identified for the set as a whole. The article suggests that the ESL students' lack of fluency results from both limitations in grammatical competency and a lack of practice in writing for varying purposes and audiences.

\section{5-301 Roache-Jameson, Sharyn, Kindergarten connections: a study of intertextuality and its links with literacy in the kindergarten classroom. Australian Journal of Language and Literacy (Norwood, Australia) 28.1 (2005), 48-66.}

Intertextuality is the human phenomenon of making connections between texts and of using an understanding of one text to interpret another. This year-long study looks at 23 kindergarten children's developing intertextual awareness in the setting of an Australian kindergarten class. The teacher-researcher video-taped the daily literacy session three times each week and also collected data on student's home literacy background, school achievement and other relevant details. Intertextual connections in the videoed literacy sessions were coded and analysed and the paper describes several such episodes in detail. Meaning in the classroom is collaboratively constructed, resulting in a community of learners sharing texts and experiences, and discovering how to make more connections between texts. The kindergarten teacher can encourage this rich literary environment by using a variety of texts and leading inquiry-based discussions.

\section{5-302 Ryan, Josephine, Young people choose: adolescents' text pleasures. Australian Journal of Language and Literacy (Norwood, Australia) 28.1 (2005), 38-54.}

This paper describes the ways in which adolescents read and interact with texts outside their formal schooling. Fifty-three students aged 14-16 in Melbourne secondary schools were interviewed and asked to discuss the print and electronic texts that they chose to read for pleasure. This revealed students' 'passion' for texts very different to those studied in school. Gender, social class and speaking a language other than English at home are discussed as factors in affecting students' choice of reading material. The author notes that at secondary-school level there is scant opportunity within school to choose what to read, with a consequent decline in reading among this age group. The paper suggests that teachers could build on students' strengths and preferences in order to keep students' interest, while recognising that to do so would entail significant curriculum change.

05-303 Rymes, Betsy (Georgia U, USA; brymes@coe.uga.edu), Contrasting zones of comfortable competence: popular culture in a phonics lesson. Linguistics and Education (Amsterdam, the Netherlands) 14.3-4 (2003), 321-335.

The contrast between the current national skills-based reading agenda in the United States and the complex forms of technology and entertainment available for children today suggests a kind of 'future shock' analogous to that identified during the rise of television in the 1960s. Adult reactions to future shock can take the form of a retreat to a comfort zone, in which one can deny that the world is changing. Children, however, bring their own familiarities with new media to the classroom, often in a way that can force adults out of this retreat, out of the role of expert and into the role of novice. This paper looks at the notion of future shock as it becomes instantiated in language and interaction in a reading lesson with second language learners. Bringing the concepts of communicative competence and language socialisation to a Vygotskian understanding of development, it suggests that current educational policy encourages teachers and students both to retreat to differing comfort zones, and that a policy which encourages them to depart from those retreats, by taking advantage of students' multiple communicative competencies, could more adequately prepare students to take active roles in the current multimedia world.

\section{5-304 Skillen, Jan \& Emily Purser (U of} Wollongong, Australia), Teaching thesis writing: policy and practice at an Australian university. Hong Kong Journal of Applied Linguistics (Hong Kong, China) 8.2 (2003), 17-33.

As an indicator of serious engagement in an academic discourse, thesis writing enjoys universal recognition. While its importance in higher education is unquestioned, the need to teach students how to write a thesis (let alone what method to use) has been less generally accepted. In Australia, explicit instruction in thesis writing was rare until quite recently, but is now widespread and becoming almost mandatory. This paper briefly explains the shift and describes how the teaching of thesis writing is approached at the University of Wollongong. The Learning Development unit supports student learning across both undergraduate and postgraduate programs and is recognised within Australia as an innovative and leading provider of quality methods and resources for instruction in language, literacy and learning in tertiary education. The paper outlines some key strategies and techniques that the unit uses to teach thesis writing and indicates the impact of its practices on students and supervisors. While critical discussion of the theoretical bases of its work is invited, the focus of this paper is on describing the unit's practices.

05-305 Stapleton, Paul (Hokkaido U, Japan; paulstapleton@gmail.com), Using the web as a research source: implications for $L 2$ academic 
writing. The Modern Language Journal (Malden, MA, USA) 89.2 (2005), 177-189.

As scholars increasingly view the World Wide Web as a legitimate research resource in academic writing, questions remain regarding how Web sources, as new media, influence the writing of second and foreign language learners. Via a 17-item questionnaire and an analysis of the references to 243 Web sources in the essays of 43 Japanese undergraduate English as a foreign language writers, this study examined whether Webbased research influenced the writers' topic choice; their choice of Web sources was appropriate for an academic essay; and using an online, electronic environment would lead writers to use new languagerelated strategies. The findings suggested a possible shift towards more internationally oriented topics as a result of the predominance of English language sites on the Web. In addition, a considerable portion of the participants' references came from Web genres of questionable suitability for an academic paper. In a similar vein, working in an electronic environment was an enticement for some L2 writers to take ethically dubious shortcuts in their writing.

\section{5-306 Starfield, Sue (U of New South Wales, Australia), The evolution of a thesis-writing course for Arts and Social Sciences students: what can applied linguistics offer? Hong Kong Journal of Applied Linguistics (Hong Kong, China) 8.2 (2003), 137-154.}

This article discusses the development of a faculty-based thesis-writing course for postgraduate Arts and Social Sciences research students at the University of New South Wales. Initially the course was conceptualised as being solely for English as a Second Language (ESL) students whose language problems were seen to be an obstacle to the writing of their theses. In 2002, it was decided to open the course to all postgraduate research students in the Faculty. The course uses annotated examples of Australian Arts and Social Sciences theses from the Australian digital theses website and focuses on the writing of the different sections of the thesis genre. The annotations draw on current applied linguistic research into academic writing which is mediated for the students into a comprehensible metalanguage. The paper discusses examples of this approach. Student responses to this pedagogy, which have been extremely positive, are also discussed.

\section{5-307 Strauss, Pat, Jo Ann Walton \&}

Suzanne Madsen (Auckland U of Technology, New Zealand), 'I don't have time to be an English teacher': supervising the EAL thesis. Hong Kong Journal of Applied Linguistics (Hong Kong, China) 8.2 (2003), 1-16.

Over the last few years the ranks of postgraduate students in New Zealand universities have been swelled by English as an Additional Language (EAL) students, both international and domestic. This has resulted in a quite dramatic change in the demands placed on postgraduate supervisors, a trend common to many universities around the world. There are many indications that this shift is not without considerable difficulties, one of which is obviously the linguistic challenges that these students face. Our study, based at a New Zealand university, explored the experiences and expectations of postgraduate supervisors who work with EAL students, and the EAL students themselves, in relation to thesis writing. In this paper we concentrate on the insights revealed by the supervisors in semistructured, in-depth interviews. We explore their views on the challenges they face as supervisors of EAL students, the extent of their responsibility as far as the language of the thesis is concerned and the role of the university in the relationship. We demonstrate that the concerns of supervisors relate to considerably more than language issues and suggest supervision may be one of the most challenging aspects of contemporary academic life.

\section{5-308 Terras, Melissa (U of London, UK;} m.terras@ucl.ac.uk), Reading the readers: modelling complex humanities processes to build cognitive systems. Literary and Linguistic Computing (Oxford, UK) 20.1 (2005), 41-59.

This paper describes the initial stages in the development of a computer system designed to aid historians in the reading of the stylus tablets. It explains how a detailed investigation was undertaken, using knowledge elicitation techniques borrowed from Artificial Intelligence, Cognitive Psychology and Computational Linguistics, to elicit the processes experts use whilst reading an ancient text. The resulting model was used as the basis of a computer architecture to construct a system which takes in images of the tablets and outputs plausible interpretations of the documents. It is demonstrated that using knowledge elicitation techniques can further the understanding of complex processes in the humanities and that these techniques can provide an underlying structure for the basis of a computer system that replicates that process. As such it provides significant insight into how experts work in the humanities, whilst providing the means to develop tools to assist them in their complex task.

05-309 Turner, Joan ( $U$ of London, UK), Writing a Ph.D. in the contemporary humanities. Hong Kong Journal of Applied Linguistics (Hong Kong, China) 8.2 (2003), 34-53.

This article is based on a case study of a Korean student writing her Ph.D. in English in the contemporary humanities, drawing particularly on post-colonial and psychoanalytic theory. It covers issues of interdisciplinarity in the contemporary humanities, how language use and rhetorical strategies are affected by the questioning of traditional epistemic norms in contemporary theorising, and comments on the role of language proficiency as well as institutional perceptions of the role of language. The tension between traditional macro-structures and 
possibilities for innovation in the genre 'Ph.D.' is discussed in the context of semi-structured interviews with two supervisors, but the suggestion is that shifts in rhetorical strategy are occurring predominantly at the micro-level.

\section{5-310 Wallace, Catherine (Institute of}

Education, UK; c.wallace@ioe.ac.uk),

Conversations around the literacy hour in a multilingual London primary school. Language and Education (Clevedon, UK) 19.4 (2005), 322-338.

This study was conducted against the background of a British government initiative - The National Literacy Strategy - which prescribes a daily hour of formal literacy instruction for primary-aged children, known as the Literacy Hour. The paper describes the developing understanding and experience of literacy of four bilingual Year Five children, studying in a multilingual London school. Conversations about literacy and the Literacy Hour with the children two boys and two girls - were recorded for one hour a week over one school year. The focus was on the impact of the Literacy Hour on the children's understanding of literacy as revealed through their personal talk about text. Conversational data are divided into four sets, moving from relatively structured, 'on task' talk, closely aligned to the Literacy Hour, to talk which embraces more widely the children's cultural and linguistic experiences, resources and attitudes. The Literacy Hour plays a relatively small part among the rich literacy resources, crossing both home and school boundaries, which the children make use of in everyday life.

05-311 Yamada, Kyoko (wsedikol@hotmail.com), Lexical patterns in the eyes of intermediate EFL readers. RELC Journal (Thousand Oaks, CA, USA) 36.2 (2005), 177-188.

Hoey (1991) claims that lexical patterns, which are a network of repetitious and synonymous words of a text, can be identified even by elementary foreign language readers. The present study investigates whether intermediate Japanese EFL readers can actually find elements of lexical patterns of a text Hoey uses in his book to demonstrate the concept of lexical patterns. Pedagogical implications of using lexical patterns in teaching reading to English language learners are also discussed.

05-312 Yamashita, Junko (Nagoya U, Japan), Reading attitudes in L1 and L2, and their influence on L2 extensive reading. Reading in a Foreign Language (Honolulu, HI, USA) 16.1 (2004), $1-19$.

The present study examines the relationship between first language (L1) and second language (L2) reading attitudes, and learners' performance in L2 extensive reading. From responses to a questionnaire by fifty- nine Japanese university students, four reading attitude variables were identified (Comfort, Anxiety, Value, Selfperception), in both L1 and L2. Results of analyses using these four variables are summarised on two levels. First, the study supports the transfer of the affective domain of reading (attitudes) from L1 to L2. But L2 proficiency does not affect this transfer in the way in which the linguistic threshold hypothesis would predict if this hypothesis were applied to the affective domain. Since this hypothesis explains the transfer of the cognitive domain of reading (i.e. reading abilities and strategies), these findings suggest that cognitive and affective domains of reading relate differently in L1 and L2. There are different degrees of transferability among different attitude variables: what learners think (Value) is more likely to transfer from L1 to L2 than what they feel (Comfort, Anxiety, Self-perception). Second, from a more pedagogical point of view, the positive feeling towards reading, both in L1 and L2, facilitates learners' performance in extensive reading. The study has thus demonstrated the importance of understanding learners' attitudes (particularly feelings) to reading both in L1 and L2 for encouraging L2 learners' extensive reading.

05-313 Zhang, Hao \& Rumjahn Hoosain (The $U$ of Minnesota, USA), Activation of themes during narrative reading. Discourse Processes (Mahwah, NJ, USA) 40.1 (2005), 57-82.

The theme of a narrative text is its main point or moral that is often implied between lines. A rapid serial visual presentation procedure (RSVP) was used to examine the online status of generation of thematic inference during narrative text comprehension. In Experiment One, the target words were presented in different contexts with different time courses. The results showed that the facilitative effects of inferential contexts were significant for target words at the RSVP rate of $400 \mathrm{~ms}$ and stimulus onset asynchrony of $600 \mathrm{~ms}$. There were no facilitative effects below this threshold. In Experiment Two, the location of target words was manipulated while maintaining the same presentation times. Further observed were facilitative effects of inferential contexts on target words appearing at the end of all the texts and at the middle of the texts when the reader had the correct expectation of the outcome in a text. These results suggested that thematic inference is normally generated online during comprehension. These findings indicate that the global theme of a narrative text is a fundamental component of text representation in reading. The result provides support for the constructionist theory of inference generation and the importance of theme information in constructing a coherent text representation.

\section{Language testing}

doi:10.1017/S0261444805242994

05-314 Alderson, J. Charles (Lancaster U, UK) \& Ari Huhta, The development of a suite of computer-based diagnostic tests based on the 
Common European Framework. Language Testing (London, UK) 22.3 (2005), 301-320.

DIALANG is an on-line language assessment system, which contains tests in 14 European languages and is based on the Common European Framework of Reference (CEFR). It is the first major testing system that is oriented towards diagnosing language skills and providing feedback to users rather than certifying their proficiency. This article describes the contents of Version 1 of DIALANG and the way in which the system works. This is followed by an account of the development of DIALANG tests and of the pilot testing and standard setting procedures. The results of the first analyses of items and self-assessment statements, and of the standard setting procedures, are reported. The article focuses on the results for English, but findings for some other languages are also touched upon.

\section{5-315 Al-Hamly, Mashael \& Christine}

Coombe (Kuwait U, Kuwait), To change or not to change: investigating the value of MCQ answer changing for Gulf Arab students. Language Testing (London, UK) 22.4 (2005), 509-531.

This study investigates whether the practice of answer changing on multiple-choice questions (MCQs) is beneficial to Gulf Arab students' overall test performance. The proficiency exam used in this study is the Michigan English Language Institute College English Test - Grammar, Cloze, Vocabulary, Reading (MELICET-GCVR), which was developed using retired forms of the Michigan English Language Assessment Battery (MELAB). This exam was administered to 286 students at Kuwait University and Dubai Men's College, Higher Colleges of Technology. Findings suggest that we should encourage students to change answers judiciously after they have scrutinised their original answers for more plausible alternatives. Most important, students should not be influenced by traditional perceptions that incorrectly malign answer changing. The effect of item difficulty as well as its impact on answer changing is also reported. Finally, information on the efficacy of one specific objective test strategy, that of changing answers, is disseminated.

05-316 Broadfoot, Patricia M. (U of Bristol, UK), Dark alleys and blind bends: testing the language of learning. Language Testing (London, UK) 22.2 (2005), 123-141.

This article presents a revised version of the author's Samuel Messick Memorial lecture in 2003. Messick's emphasis on consequential validity is used to identify some of the negative effects of current practices as they affect the quality of students' learning and motivation. It suggests that there have been many 'dark alleys' in which poorly understood assessment technologies have been used thoughtlessly or have been located on 'blind bends' in which a lack of sufficient preparation and knowledge has resulted in assessment practices that are dangerous and, potentially, very damaging to learners. In the light of these problems the article argues for a more constructive approach to assessment. Taking the particular context of language learning, the article describes a new assessment tool, The Effective Lifelong Learning Inventory (ELLI), which has a very different purpose: the identification of an individual's power to learn. It argues the need to replace the conventional lexicon of testing with a focus on key learning dimensions such as confidence, collaboration, critical curiosity and creativity - the feelings and dispositions that are argued to be central to learning.

05-317 Cumming, Alister ( $U$ of Toronto, Canada; acumming@oise.utoronto.ca), Robert Kantor, Kyoko Baba, Usman Erdosy, Keanre Eouanzoui \& Mark James, Differences in written discourse in independent and integrated prototype tasks for next generation TOEFL. Assessing Writing (Amsterdam, the Netherlands) 10.1 (2005), 5-43.

The authors assessed whether and how the discourse written for prototype integrated tasks (involving writing in response to print or audio source texts) field tested for Next Generation TOEFL ${ }^{\circledR}$ differs from the discourse written for independent essays (i.e. the TOEFL Essay ${ }^{\circledR}$ ). 216 compositions written for six tasks by 36 examinees in a field test - representing score levels 3,4 and 5 on the TOEFL Essay - were selected and then coded for lexical and syntactic complexity, grammatical accuracy, argument structure, orientations to evidence and verbatim uses of source text. Analyses with nonparametric MANOVAs followed a three (task type: TOEFL Essay, writing in response to a reading passage, writing in response to a listening passage) by three (English proficiency level: score levels 3, 4 and 5 on the TOEFL Essay) within-subjects factorial design. The discourse produced for the integrated writing tasks differed significantly from the discourse produced in the independent essay for the variables of: lexical complexity, syntactic complexity, rhetoric and pragmatics. Across the three English proficiency levels, significant differences appeared for the variables of grammatical accuracy as well as all indicators of lexical complexity, one indicator of syntactic complexity (words per $T$-unit), one rhetorical aspect (quality of claims in argument structure) and two pragmatic aspects (expression of self as voice, messages phrased as summaries).

05-318 Eckes, Thomas (TestDaF Institute, the Netherlands), Melanie Ellis, Vita Kalnberzina, Karmen Piorn, Claude Springer, Krisztina Szollás \& Constance Tsagari, Progress and problems in reforming public language examinations in Europe: cameos from the Baltic States, Greece, Hungary, Poland, Slovenia, France and

Germany. Language Testing (London,UK) 22.3 (2005), 355-377.

Contributions from seven European countries pinpoint major projects, problems and prospects of reforming 
public language assessment procedures. Each country has faced unique problems in the reform process, yet there have also been several common themes emerging, such as a focus on multilingualism, communicative skills, standardisation, reference to the CEFR (Common European Framework of Reference for Languages) and certification. Future work needs to develop these themes further and to study impact and support issues as well.

\section{5-319 Figueras, Neus (Department of Education, Generalitat de Catalunya, Spain), Brian North, Sauli Takala, Norman Verhelst \& Piet Van Avermaet, Relating examinations to the Common European Framework: a manual. Language Testing (London, UK) 22.3 (2005), 261-279.}

This article deals with the linking of examinations to the Common European Framework of Reference (CEFR). Following the rapid adoption of the CEFR by the Council of Europe and the European Union as well as many national and local actors, there have been calls for guidance on how examinations could be made more transparent by means of the CEFR, especially by using its calibrated scales as a tool. The Council of Europe has recently produced a preliminary pilot Manual, which presents a procedure for doing this. The article describes the political and educational context of this work and presents the main procedures proposed in the Manual. Since the Manual is a preliminary document for discussion and feedback, the authors of the Manual join the discussion by presenting some critical reflections of their own. The article concludes by outlining two scenarios: one which predicts positive developments and the other which foresees less desirable consequences.

\section{5-320 Green, Anthony (Cambridge ESOL Examinations, Cambridge, UK), EAP study recommendations and score gains on the IELTS Academic Writing test. Assessing Writing} (Amsterdam, the Netherlands) 10.1 (2005), 44-60.

The IELTS (International English Language Testing System) test is widely accepted by university admissions offices as evidence of English language ability. The test is also used to guide decisions about the amount of language study required for students to satisfy admissions requirements. Guidelines currently published by the British Association of Lecturers in English for Academic Purposes (BALEAP) suggesting that two months of intensive English study is equivalent to one band on the nine-band IELTS scale have, in the face of changes in the international student population, recently come under scrutiny. This paper reviews recent research relating to score gains on the IELTS test and reports on two linked studies of gains made on the Academic Writing. Phase 1 involved 15,380 candidates taking the official test on two occasions and phase 2,476 learners on English for academic purposes (EAP) courses taking the IELTS
Writing test at course entry and exit. The findings call into question the basis for the current guidelines. Initial scores prove to be a stronger predictor of outcomes than course length.

05-321 Green, Rita \& Dianne Wall (Lancaster U, UK), Language testing in the military: problems, politics and progress. Language Testing (London,UK) 22.3 (2005), 379-398.

There appears to be little literature available - either descriptive or research-related - on language testing in the military. This form of specific purposes assessment affects both military personnel and civilians working within the military structure in terms of posting, promotion and remuneration, and it could be argued that it has serious social consequences if not carried out professionally and to the highest standard. This article provides a general overview of the language testing that is carried out by military teams in Central and Eastern Europe, using the findings of three surveys conducted with teams in this region. It explores the design problems the teams have had to deal with, the political issues that influence the work they do and the progress they have been able to make over recent years. The article concludes with an attempt to link these findings to broader issues in the assessment of English for Specific Purposes.

\section{5-322 Hasselgreen, Angela (The $U$ of Bergen,} Norway), Assessing the language of young learners. Language Testing (London,UK) 22.3 (2005), 337-354.

This article focuses on the plight of children as young language learners (YLLs), in the context of today's Europe. By reference to the Council of Europe's recommendations and its material in the form of the Common European Framework of Reference (CEFR) and the European Language Portfolio (ELP), questions are posed here as to how far the special needs of YLLs are being catered for by assessment practices in European schools. Examples are given of how the CEFR and ELP are currently used in YLL assessment, with a focus on recent developments in this direction in Norway, and conclusions are drawn on the outlook for YLL assessment in present day Europe.

\section{5-323 Klein, Joseph (kleinj@mail.biu.ac.il) \& David Taub, The effect of variations in handwriting and print on evaluation of student essays. Assessing Writing (Amsterdam, the Netherlands) 10.2 (2005), 134-148.}

The variety of factors contributing to the quality of handwriting or print provides an opportunity to determine the extent to which teachers are influenced by the interaction between different bias elements during evaluation of examinations. Fifty-three teachers evaluated compositions that had been awarded grades of $80 \%$ by impartial experts. A comparison was made 
of scores assigned to essays containing a variety of biasing factors: legibility as opposed to illegibility, use of different writing tools and employment of aesthetic devices such as colour or underlining. Findings: the hypothesis examined was supported, indicating that assessors cumulatively incorporated biases deemed pertinent and fair into the grade, and overlooked those considered irrelevant.

05-324 Little, David (Trinity College, Dublin, Ireland), The Common European Framework and the European Language Portfolio: involving learners and their judgements in the assessment process. Language Testing (London, UK) 22.3 (2005), 321-336.

Learner-centred approaches to language teaching, especially those that seek to develop learner autonomy, require the learner to take decisions concerning the goals, content and methods of learning; they also assign a central role to self-assessment. Although the logic of learner-centredness demands that learner selfassessment should somehow be integrated with other forms of assessment, to date this has been only a minority concern, usually in relation to one or another form of portfolio learning. The recent publication of the Common European Framework of Reference for Languages (CEFR) and the increasingly widespread adoption of its companion piece, the European Language Portfolio (ELP), renew the challenge to develop a culture of assessment that both facilitates and takes full account of learner self-assessment. This article begins by briefly considering the importance and limitations of self-assessment in second language learning. It goes on to address issues of principle raised in turn by the CEFR and the ELP, and then reports on a project that (i) has drawn on the CEFR to define an ESL curriculum for non-English-speaking pupils attending Irish primary schools; (ii) has developed a version of the ELP as the foundation of teaching and learning; and (iii) is currently elaborating assessment and reporting procedures in which learner self-assessment plays a central role.

\section{5-325 Lumley, Tom \& Barry O'Sullivan} (Australian Council for Educational Research, Australia), The effect of test-taker gender, audience and topic on task performance in tape-mediated assessment of speaking. Language Testing (London,UK) 22.4 (2005), 415-437.

Performance in tests of spoken language can be influenced by a wide range of features of both task and participants. This article hypothesises that there may be effects on performance attributable to an interaction of variables such as the task topic, the gender of the person presenting the topic and the gender of the candidate. In contrast to previous studies, which have examined speaking tests involving face-to-face interaction, this study considered the issue in a tape-mediated test delivered in a language laboratory, with no interlocutor present, but where stimulus material is presented by one or more speakers, one of whom acts as 'audience' for the candidate's speech. The test was taken by 894 students graduating from Hong Kong universities. In an advice-giving task, the last of a series involving different situations and audiences, topics considered stereotypically 'male'-oriented or 'female'-oriented were presented with systematic changes in the roles taken by the male and female speakers. A multi-faceted Rasch analysis examined the interaction of test-taker gender, task topic and gender of presenter/audience. The results showed small effects for some, but not all, of the hypothesised interactions. Examples of differential performance by male and female candidates on other tasks are also presented. The article concludes with discussion of the implications for task design and test content.

\section{5-326 Luxia, Qi (Guandong U of Foreign}

Studies, China), Stakeholders' conflicting aims undermine the washback function of a high-stakes test. Language Testing (London, UK) 22.2 (2005), 142-173.

High-stakes tests are often used as agents for change, but 'attempts to introduce change in the classroom are often not as effective as their designers hoped they would be' (Wall 1996: 334). The National Matriculation English Test (NMET) in China is such a case. Apart from its primary function of selecting candidates for institutions of higher education, the NMET was designed specifically to promote changes in ELT (English language teaching) in schools. Unfortunately, it has fallen short of the goal. This study set out to examine the reasons why the NMET failed to bring about the intended changes or washback effects. For this purpose, data were collected through interview and questionnaire from eight NMET constructors, six English inspectors, 388 teachers and 986 students. The results show that the most important reason for the test failing to achieve the intended washback is that its two major functions - the selection function and the function of promoting change - are in many ways in conflict with each other, making it a powerful trigger for teaching to the test but an ineffective agent for changing teaching and learning in the way intended by its constructors and the policymakers.

\section{5-327 Poehner, Matthew E. \& James P.} Lantolf (The Pennsylvania State U, USA), Dynamic assessment in the language classroom.

Language Teaching Research (London, UK) 9.3 (2005), 233-265.

The focus of this paper is on the implementation of Dynamic Assessment (DA) in the L2 classroom setting. DA is an approach to assessment and instruction derived from Vygotsky's theory of the Zone of Proximal Development (ZPD). In what follows, we will first discuss briefly the concept of the ZPD and its realisation 
in DA procedures. Next we will briefly discuss the work of Reuven Feuerstein, whose work on DA is most directly relevant to the goal of the present article. Finally, we will compare DA to Formative Assessment (FA) and will suggest how FA might be reconceptualised according to DA principles. In the language testing literature, FA is usually contrasted with Summative Assessment on the grounds that the former is intended to feed back into the teaching and learning process while the latter reports on the outcomes of learning. Moreover, FA procedures are generally considered to be less systematic and not as high-stakes as summative assessments. Following ReaDickins \& Gardner (2000), however, we suggest that FA is not necessarily low-stakes and that it can be carried out quite systematically, yielding results that may be more systematic and revealing with regard to learner development than summative assessments.

\section{5-328 Stansfield, Charles W. \& William E. Hewitt (Second Language Testing Inc., USA), Examining the predictive validity of a screening test for court interpreters. Language Testing (London, UK) 22.4 (2005), 438-462.}

The United States Court Interpreters Act (US Congress, 1978) requires that interpreters in US federal courts be certified through a criterion-referenced performance test. The Federal Court Interpreter Certification Examination (FCICE) is a two-phase certification battery for federal court interpreters. Phase I is a multiple-choice Written Examination (WE) used to screen candidates for eligibility to take the Phase II criterion-referenced Oral Examination (OE). This study evaluates the predictive validity of the WE in relationship to examinee performance on the OE, using an experimental sample of examinees who took the tests concurrently. Results indicate that the WE correlates appropriately with performance on the OE (.648 and .676 for the English and Spanish subtests, respectively). Nearly all of those who failed the WE also failed the OE, although several false negatives were identified. However, when the data were reanalysed using a lower cut score for the Phase I WE, nearly all false negatives were eliminated. A follow-up study replicated the findings with a different sample. The authors discuss the advantages and disadvantages of lowering the passing score on the screening test in light of their findings. The study and subsequent discussion provide an example of how an established passing score can be reconsidered and modified.

\section{5-329 Trites, Latricia (Murray State U, USA) \& Mary McGroarty, Reading to learn and reading to integrate: new tasks for reading comprehension tests? Language Testing (London, UK) 22.2 (2005), 174-210.}

To address the concern that most traditional reading comprehension tests only measure basic comprehension, this study designed measures to assess more complex reading tasks: Reading to Learn and Reading to
Integrate. The new measures were taken by 251 participants: 105 undergraduatenative speakers of English, 106 undergraduate non-native speakers and 40 graduate non-native speakers. The research subproblems included determination of the influence of overall basic reading comprehension level, native language background, medium of presentation, level of education and computer familiarity on Reading to Learn and Reading to Integrate measures; and the relationships among measures of Basic Comprehension, Reading to Learn, and Reading to Integrate. Results revealed that native language background and level of education had a significant effect on performance on bothexperimental measures, while other independent variables did not. While all reading measures showed some correlation, Reading to Learn and Reading to Integrate had lower correlations with Basic Comprehension, suggesting a possible distinction between Basic Comprehension and the new measures.

05-330 Uiterwijk, Henny (Citogroep, Arnem, the Netherlands) \& Ton Vallen, Linguistic sources of item bias for second generation immigrants in Dutch tests. Language Testing (London, UK) 22.2 (2005), 211-234.

This article reports the first results of a long-term research project focusing on the detection and possible linguistic causes of differential item functioning (DIF) for second generation immigrant students in the Final Test of Primary Education in the Netherlands. The main aim of the project is to provide test constructors with information which is as detailed as possible about sources of DIF in order to help them avoid item bias in future forms of the test. The project was carried out in three steps. First, DIF items were identified using two statistical procedures: IRT and Mantel-Haenszel. The second stage was an investigation of sources of DIF, beginning with an inventory of subject matter elements which can cause difficulty for immigrant students, according to previous educational research. This was followed by a careful evaluation of DIF items by the researchers and by external experts, and a think-aloud experiment with students. At the third stage, judges decided whether each DIF item was biased and thus reduced the construct validity of the test. The article concentrates on the classification of linguistic sources of DIF and a consideration of whether they work to the advantage or disadvantage of immigrant students.

05-331 Weems, Gail H. (Arkansas Little Rock U, USA; ghweems@ualr.edu), Anthony J.

Onwuegbuzie \& Daniel Lustig, Profiles of respondents who respond inconsistently to positively- and negatively-worded items on rating scales. Evaluation \& Research in Education (Clevedon, UK) 17.1 (2003), 45-60.

Many instruments, especially Likert-type scales, contain both positively- and negatively-worded items within the same scale (i.e. mixed-item format). A major reason for this practice appears to be to discourage 
response sets from emerging. Using this format also helps the analyst to detect response sets that occur in data sets and thus eliminate them from subsequent analyses. However, some psychometricians seriously question the use of mixed-item formats, positing that positively- and negatively-worded items within a scale are not measuring the same underlying trait. Limited evidence has suggested that certain individuals are more predisposed to providing differential response patterns when responding to a mixed-item format scale. However, to date, only a few characteristics of these differential-responding individuals have been identified. In this study, the researchers analysed responses to several scales utilising mixed-item formats. For example, on canonical correlation analysis, a sample of 185 students revealed a relationship between degree of differential responses between positively- and negatively-worded items on three six-item measures of foreign language anxiety (i.e. input, processing and output anxiety) and several dimensions of self-perception, study skills and locus of control.

\section{5-332 Weir, Cyril J. (Roehampton U, UK), Limitations of the Common European Framework for developing comparable examinations and tests. Language Testing (London, UK) 22.3 (2005), 281-300.}

The Common European Framework of Reference (CEFR) posits six levels of proficiency and defines these largely in relation to empirically derived difficulty estimates based on stakeholder perceptions of what language functions expressed by 'Can-do' statements can be successfully performed at each level. Though also containing much valuable information on language proficiency and advice for practitioners, in its present form the CEFR is not sufficiently comprehensive, coherent or transparent for uncritical use in language testing. First, the descriptor scales take insufficient account of how variation in terms of contextual parameters may affect performances by raising or lowering the actual difficulty level of carrying out the target 'Can-do' statement. In addition, a test's theorybased validity - a function of the processing involved in carrying out these 'Can-do' statements - must also be addressed by any specification on which a test is based. Failure to explicate such context and theorybased validity parameters - i.e. to comprehensively define the construct to be tested - vitiates current attempts to use the CEFR as the basis for developing comparable test forms within and across languages and levels, and hampers attempts to link separate assessments, particularly through social moderation.

05-333 Xi, Xiaoming ( $U$ of California, USA), Do visual chunks and planning impact performance on the graph description task in the SPEAK exam? Language Testing (London, UK) 22.4 (2005), 463-508.

This study examines how task characteristics (the number of visual chunks and the amount of planning time) and test-taker characteristics (graph familiarity) influence the perceptual and cognitive processes involved in graph comprehension, the strategies used in describing graphs, and the scores obtained on the graph description task in a semi-direct oral test. Specifically, it investigates whether providing planning time and reducing the number of visual chunks in bar and line graphs help mitigate the influence of graph familiarity on language performance on graph description tasks. These research questions were explored using a structural equation modeling approach in a framework of the influence of the interaction between task characteristics and test-taker characteristics on task performance. It was found that graph familiarity affected the overall communicative quality of participants' performance on these graph description tasks. Graph familiarity thus represented a potential source of constructirrelevant variance. However, reducing the number of visual chunks in a graph and providing planning time positively impacted the perceptual and cognitive processes involved in graph comprehension and helped mitigate the influence of graph familiarity. Practical and theoretical implications of this study are discussed.

\section{Teacher education}

doi:10.1017/S0261444805252990

\section{5-334 Angelides, Panayiotis, Maria Evangelou \& James Leigh (Intercollege, Cyprus), Implementing a collaborative model of action research for teacher development. Educational Action Research (Oxford, UK), 13.2 (2005),} 275-290.

In this article the authors develop and implement a collaborative model of action research. An academic and a teacher collaborate with the purpose of improving the teaching practice and the teaching abilities of the teacher in mixed ability classes, and the collaborative ability of both academic and teacher. After a year of collaboration certain themes, related to the improvement of the teacher's practice, emerged. Specifically, the teacher developed a larger repertoire of teaching techniques and began to develop collaborations with other teachers and 'outsiders'. She also developed techniques for investigating her practice and was better prepared for her lessons. Generally, in her classroom, more authentic relationships were developed.

05-335 Brock, Cynthia, Lori Helman \& Chitlada Patchen ( $U$ of Nevada, USA), Learning to conduct teacher research: exploring the development of mediated understandings. Teachers and Teaching: Theory and Practice (Abingdon, UK) 11.1 (2005), 73-94.

There is a growing trend in education to advocate for the inclusion of teacher researcher voices in local, state, national and international conversations about 
classroom teaching and learning. While many scholars agree that teacher researchers can, do, and should make important contributions to the educational research community, much remains to be learned about how teachers learn to engage in quality reflective inquiry. Our goal in this work is to explore instances of one practicing teacher's opportunities to learn to conduct teacher research. The practicing teacher (pseudonym, Dot) was enrolled in a university seminar that focused on learning to conduct classroom research. For this qualitative investigation we analysed field notes taken during 13 class sessions across the semester, audiotapes of classroom interactions during class sessions, and artefacts such as written class reflections about course topics and drafts of course assignments. Results revealed that a complex set of significant instances of Dot's learning about teacher research shaped both what and how she learned. The careful descriptions of Dot's instances of learning can inform individual instructors about moment-to-moment instructional decisions when working with teachers in teacher research courses. Additionally, this work can inform a broader educational community with respect to how classes pertaining to teacher research might be reconceptualised to promote systematic research of one's practice as a 'way of being' as a classroom teacher.

05-336 Burdelski, Matthew (California U, USA; mburdel@ucla.edu), Close- and open-ended narratives of personal experience: weekly meetings among a supervisor and teaching assistants of a 'Japanese language education practicum'. Linguistics and Education

(Amsterdam, the Netherlands) 15.1-2 (2004), 3-32.

In comparison to other forms of talk in interaction, narrative is a temporal and logical ordering of events, driven by a need to establish coherence across past, present and future experience. Despite research on narrative in everyday conversation, teacher education has not necessarily taken multi-party interaction as a primary locus of analysis. An assumption has been that narrative primarily resides within an individual author, as illustrated through written questionnaires and interviews with a researcher or facilitator who provides a relatively passive audience. As a result, teacher education lacks a clear understanding of narrative as an interactive process, embedded within situated talk and activity, and its relationship to professional development. The present paper addresses these issues by examining narratives of personal experience as they emerged within supervisor-teaching assistant talk during weekly meetings. The findings reveal that the supervisor played a crucial role in narrative discourse, using her own close-ended narratives to justify a position or make suggestions for future courses of action by the group, and giving meaning to open-ended narratives in written journals and group discussion. The implications suggest that structured, iterative reflection with a supervisor (and peer group) focusing on specific issues and goals contributes to professional development.
05-337 Dooly, Melinda (U of Barcelona, Spain), How aware are they? Research into teachers' attitudes about linguistic diversity. Language Awareness (Clevedon, UK) 14.2/3 (2005), 97-112.

This paper reports research designed to highlight how three groups of teachers make sense of linguistic, ethnic and cultural diversity in the classroom. The collecting of the data concentrated on the way in which the participants in the study worked together to construct socially negotiated meanings of linguistic diversity within the context of language learning, principally in the English as a Foreign Language classroom. A key assumption of the analysis is that the speakers within a conversation 'construct' or 'assemble' categories which are based on a background of 'common knowledge', and that the assembly of categories are not descriptions of truth; rather they are the speakers' constructed versions of how they make sense of the world. The research indicated that the participants in this study consistently assembled the category of 'diversity' with features such as 'problematic', 'difficult', 'hard'; thus implying that there was a common understanding of this type of classroom. However, the analysis indicates that, despite an overall similarity among the groups' categorisations concerning linguistic diversity, the preservice teachers were more likely to re-negotiate their initial categories towards more positive understandings of linguistic diversity.

\section{5-338 Ezer, Hanna (Levinsky College of Education, Tel-Aviv, Israel; hezer@macam.ac.il) \& \\ Tamar Sivan, 'Good' academic writing in Hebrew: the perceptions of pre-service teachers and their instructors. Assessing Writing}

(Amsterdam, the Netherlands) 10.2 (2005),

117-133.

This study explores the perceptions of different groups of L1 and L2 Israeli pre-service teachers and their instructors regarding GooDacademic writing. The study further investigates whether those perceptions accurately reflect their knowledge of academic writing. Eighty-one participants graded three different essays written by immigrant students and provided written explanations of their assessments. An unstructured oral interview followed. Results indicated that guidelines and instruction in academic writing enabled students to accommodate their instructor's demands and to assess academic text. Although no significant difference was found between the students' assessments and the instructors' actual assessments, students perceived the instructors' assessments to be stricter than their own. Nevertheless, the perception of academic literacy was not consistent among the various groups. IsraeliArab students viewed the language as an instrument for integration into the mainstream of Israeli society and thus produced stricter assessments. They believed that instructors were not strict enough. Immigrant students, relying on their cognitive abilities and previous knowledge of academic standards were familiar with 
the literacy discourse and the instructors' expectations. They believed that even if an essay were good, the instructors would apply stricter standards.

\section{5-339 Hayes, David (U of Birmingham, UK), Exploring the lives of non-native speaking English educators in Sri Lanka. Teachers and Teaching: Theory and Practice (Abingdon, UK) 11.2 (2005), 169-194.}

This paper uses in-depth interview data to explore aspects of the lives of non-native speaking English educators working in the state education system in Sri Lanka. In so doing the research focus is on the educators themselves and the paper discusses such issues as careers as English teachers - motives for entering teaching, career progression, becoming a teacher educator; professional self-development; beliefs about teaching, learning and teacher education; and the wider socioeducational context of English teaching in Sri Lanka. The paper offers insights into the development of non-native speaking TESOL teachers by highlighting certain features of practice in particular contexts and by providing 'snapshots' of teaching and learning in state systems beyond the western world which may inform thinking more broadly about international English language teaching and learning.

\section{5-340 Korthagen, Fred (Utrecht U, the} Netherlands) \& Angelo Vasalos, Levels in reflection: core reflection as a means to enhance professional growth. Teachers and Teaching: Theory and Practice (Abingdon, UK) 11.1 (2005), 47-71.

Reflection is currently a key concept in teacher education. The reflection process is often described in terms of a cyclical model. In the present article, we explain how such a model can be used for supporting student teachers' reflection on practical situations they are confronted with, and on their behaviour, skills and beliefs in such situations. In some cases, however, more fundamental issues appear to influence teachers' practical functioning. For example, their self-concept can have a decisive influence on the way they function, or they may do what is expected of them, and yet not feel truly involved. In such cases, a more fundamental form of reflection is needed, which in this article we refer to as 'core reflection'. The focus on core reflection concurs with the recent emphasis in psychology on attending to people's strengths rather than their deficiencies.

05-341 Lynch, Brian (Portland State U, USA; bklynch@pdx.edu) \& Peter Shaw, Portfolios, power, and ethics. TESOL Quarterly (Alexandria, VA, USA) 39.2, 263-298.

Portfolios have been used in a variety of ways for assessing student work. In education, generally, and more specifically in second language education, portfolios have been associated with alternative assessment (Wolf, Bixby, Glenn \& Gardener 1991, Darling-Hammond 1994, Hamayan 1995, Shohamy 1996). This article defines ALTERNATIVE ASSESSMENT as representing a paradigm and culture that is different from traditional testing, requiring a different approach to addressing the issues of validity and ethics. We present a framework that integrates a consideration of how power relations determine the ethics and validity of assessment inferences. We then apply that framework to the assessment of student portfolios in a master of arts in TESOL (MA TESOL) program.

\section{5-342 Roulston, Kathryn, Roy Legette, Monica DeLoach \& Celeste Buckhalter Pittman (U of Georgia, USA), What is 'research' for teacher-researchers? Educational Action Research (Oxford, UK) 13.2 (2005), 169-190.}

'Practitioner research' or 'teacher-research' has been widely promoted as a way of facilitating professional development for pre-service and in-service teachers, promoting change and reform in $\mathrm{K}-12$ settings, and giving voice to teachers' personal and professional knowledge. In this article, the authors consider what 'research' is for teacher-researchers. They argue that varying interpretations of definitions of research may exclude practitioners from the production of researchbased knowledge and hinder teacher-research collaborations among university educators and K-12 teachers. They view this exclusion as part of the larger 'knowledge' critique of teacher-research. The article first reviews contrasting definitions of 'research,' before exploring how interpretations of research definitions proved problematic within the context of their teacherresearch group. The authors propose that the current emphasis in the USA on forms of 'research' that focus on causal questions to examine evidence of effectiveness, overlooks questions and issues central to teachers' work, and re-inscribes the boundaries between 'research' and 'practice' that have traditionally divided university researchers and K-12 practitioners. Such narrow definitions of research, they argue, discourage practitioners from engaging in teacher-research, and prohibit the development of a practitioner-based stockof-knowledge grounded in teachers' knowledge and experience.

05-343 Santagata, Rossella (California U, USA rossellas@lessonlab.com), ‘Are you joking or are you sleeping?': cultural beliefs and practices in Italian and U.S. teachers' mistake-handling strategies. Linguistics and Education (Amsterdam, the Netherlands) 15.1-2 (2004), 141-164.

This study investigates the role of cultural beliefs and practices in teaching. The focus is the process of socialisation by which students learn to deal with mistakes. The study is based on an analysis of teacherstudent interactions surrounding mistakes videotaped in 30 Italian and 30 US eighth-grade mathematics lessons. 
The video recordings are complemented by interviews with teachers and students and videotapes of elementary school classes. The study combines discourse analytic approaches with quantification of some aspects of the interactions observed. Italian and US students have different experiences with mistakes that largely depend on how teachers organise instructional activities and frame classroom participant roles. Mistakes are discussed publicly in Italy twice as often as in the United States. US teachers mitigate their responses; Italian teachers aggravate them. Mistake-handling activities may be understood as culturally specific interplays of beliefs and practices. The complexity of these interplays, it is suggested, is a significant obstacle to effective teaching innovation.

05-344 Waters, Alan (Lancaster U, UK; A.Waters@lancaster.ac.uk), Ma. Luz. C. Vilcheseo, Managing innovation in language education: a course for ELT change agents. RELC Journal (Thousand Oaks, CA, USA) 36.2 (2005), 117-136.

As a steady stream of recent papers indicates, ELT curriculum reform projects are not always as successful as they might be. One overall reason for this situation appears to be a failure to adequately take into account concepts and practices from the world of innovation management. This paper describes an attempt to contribute towards ameliorating this problem by detailing the content, activities, learning processes and outcomes of a short in-service training course on managing innovation in language education, delivered 'on-site' to a cross-section of change agents involved in a major educational reform initiative currently being undertaken in the Philippines. Data from the course are used to throw light on its value as an innovation management awareness-raising vehicle. Although illustrated in terms of a particular innovation context, the course is felt to be also of potential relevance to similar situations elsewhere.

\section{Bilingual education \& bilingualism}

doi:10.1017/S0261444805262997

05-345 Byon, Andrew Sangpil (Albany U, USA; abyon@albany.edu), Classroom assessment tools and students' affective stances: KFL classroom settings. Language and Education (Clevedon, UK) 19.3 (2005), 173-193.

The growth of KFL (Korean as a Foreign Language) programmes in the US college setting has been truly remarkable in the last three decades. However, despite the gradual and steady growth of the non-heritage student population, the predominant group has been heritage students in most KFL programmes. In addition, teaching these two groups of students effectively has been a key concern for KFL teachers and administrators. The goal of this paper is to report the use of CATs
(Classroom Assessment Techniques) as a possible pedagogical solution in dealing with this complicated issue. The paper visits the current pedagogical and administrative issues, dealing with the two student groups of KFL programmes in the US college setting, and suggests the use of CATs as a possible solution for the matter, in particular, its beneficial merits in enhancing affective stances of students. The paper argues that CATs can be valuable tools in most teaching contexts, especially for the class with mixed student composition. Although the cases presented in this paper are from a third-year Advanced Korean course, the techniques presented may be adapted for use in nearly any foreign language course that has struggled with the heritage and non-heritage student issues.

\section{5-346 Costa, Jennifer, Gary McPhail, Janet Smith \& Maria Estela Brisk (Boston College, USA), Faculty first - the challenge of infusing the teacher education curriculum with scholarship on English language learners.}

Journal of Teacher Education (Thousand Oaks, CA, USA) 56.2 (2005), 104-118.

The number of pupils who are English language learners (ELLs) in the United States has increased hugely in the last five years, creating a need for all teachers to be competent in teaching bilingual pupils in their classes. However, most teachers have little or no training in working with ELLs and changes are needed in teacher education (TE) programs to improve on this situation. This paper describes the process of change in one faculty institute. Participants included TE faculty, representatives from public schools and TE doctoral students, who were led by an expert on bilingual education. Each participant was expected to look for ways to change his or her syllabus within TE. One significant recognition from several participants was that frequently matters of ELL and multicultural education were relegated to the end of a course rather than taking a central position. Future stages of the project are designed to reach most TE faculty and raise awareness about ELLs across the TE curriculum.

05-347 Gebhard, Meg (U of Massachusetts, Amherst, USA; gebhard@educ.umass.edu), School reform, hybrid discourses, and second language literacies. TESOL Quarterly (Alexandria, VA, USA) 39.2, 187-210.

This article analyses how school reforms in the United States during the 1990s supported transformative literacy practices in the context of a Hmong-English third grade classroom. Using methods that allow for an analysis of both macro and micro discourses shaping the literacy practices of English language learners, this two-year study illustrates how combined discourses at the state, district, school, and classroom levels created a discursive space that allowed for the production of hybrid texts that disrupted, if only temporarily, many of the reproductive forces associated with modern 
schooling. Using a critical perspective of language and social change (e.g. Fairclough 1992), the author presents an analysis of texts produced and interpreted by participants in a classroom shaped by a statewide school reform initiative known as Senate Bill 1274, California's school restructuring initiative. An analysis of these texts reveals that they afforded language learners opportunities to display multilingual and multicultural identities and to appropriate academic uses of English.

05-348 Jessner, Ulrike ( $U$ of Innsbruck, Austria), Multilingual metalanguage, or the way multilinguals talk about their languages.

Language Awareness (Clevedon, UK) 14.1 (2005), 56-69.

The increase of multilingualism in both natural and formal contexts has inspired a number of studies which have concentrated on providing evidence for multilingual processing and finding out about the differences and the similarities between second and third language learning. This paper deals with the use of metalanguage in multilingual students in an introspective study of their problem-solving behaviour in lexical search. The study shows that the multilingual students make use of metalanguage in languages other than the target language during the production process. Furthermore metalanguage was found to have several functions when preceding switches and thus a control function in multilingual processing was identified. A qualitative analysis of the individual use of metalanguage turned out to support the tentative results. From the tendencies found in this study it can be concluded that investigations of metalanguage might form a valuable methodological tool for further research on the roles of, and relationship between, a multilingual's languages.

\section{5-349 Liebscher, Grit (Waterloo U, Canada;} gliebsch@uwaterloo.ca) \& Jennifer Dailey-O'Cain, Learner code-switching in the content-based foreign language classroom. The Modern Language Journal (Malden, MA, USA) 89.2 (2005), 234-247.

Using a framework based on conversation analysis, this article presents an analysis of learner code-switching between first language (L1) and second language (L2) in an advanced foreign language (FL) classroom. It was found that students code-switch not only as a fallback method when their knowledge of the L2 fails them, or for other participant-related functions, but also for discourse-related functions that contextualise the interactional meaning of their utterances. These uses strikingly resemble code-switching patterns in nonclassroom bilingual settings and show that language learners are able to conceptualise the classroom as a bilingual space. Learners orient to the classroom as a community of practice through their code-switching patterns as manifestations of a shared understanding about their actions and about themselves as members of that community.

\section{Sociolinguistics}

doi:10.1017/S0261444805272993

05-350 Biesenbach-Lucas, Sigrun (American U; sblucas@american.edu), Communication topics and strategies in e-mail consultation: comparison between American and international university students. Language Learning \& Technology (Hawaii, Manoa, USA) 9.2 (2005), 24-46.

While research has investigated the purposes for which university students communicate with their instructors via email, little research has examined differences in its use between American and international students. Research has also found that students respond favorably to using email as an additional means of communication in their courses and indicate that it may improve teacher-student communication and student learning. This article describes how email messages sent by American and international students enrolled in a teacher-preparation program to their professor were collected over the course of one semester. The messages were examined for three major communication topics (facilitative, substantive, relational) and communication strategies (requesting, negotiating, reporting). Results indicate quantitative and qualitative differences in American and international students' email topics and strategies, suggesting, similar to findings for face-to-face academic tutorials, that American students demonstrate more ability to adapt to the temporal remoteness between interlocutors in email interaction, especially when using email to set up face-to-face appointments and input on projects. One of the findings is that messages from both groups of students contained substantial relational communication, in an attempt to compensate for the lack of visual and paralinguistic clues in email.

\section{5-351 Giles, Howard \& Dorjee Dorjee ( $U$ of California, USA), Cultural identity in Tibetan}

diasporas. Journal of Multilingual and Multicultural Development (Clevedon, UK) 26.2 (2005), 138-157.

Tibetan civilisation is over two millenniums old and, today, its struggles in diaspora open up a new chapter in Tibetan history. Diasporic Tibetans (e.g. in India and the USA) have made tremendous efforts over the last few decades to maintain their way of life. This paper focuses on cultural identity in the Tibetan diasporas, with special attention focused on its historical construction as well as its two fundamental dimensions - Tibetan Buddhism and the language. Through this analysis, the authors aim to ferment a new scholarly interest in the survival of Tibetan culture among multilingual and multicultural scholars.

05-352 Kheimets, Nina G. \& Alek D. Epstein (Bar-Ilan $U$, Israel), Languages of science in the era of nation-state formation: the Israeli universities and their (non)participation in the 
revival of Hebrew. Journal of Multilingual and Multicultural Development (Clevedon, UK) 26.1 (2005), 12-36.

This paper presents sociological analysis of the linguistic and cultural identity of two of Israel's most influential and high-ranking universities during their formative years, that were also the de facto formative years of the Israeli state-in-the-making (1924-1948). It is argued that the influence of external universal factors on a nation-state was sometimes crucial long before the period characterised by social scientists as an era of globalisation. Influenced by European nationalism, the leaders of the Zionist movement emphasised the importance of the restoration of Hebrew as a national language. In various European national movements the universities played a central role in the revival or creation of a national culture: the language, the national epic, the folklore were all cultivated and nurtured by the universities. This was not the case in the Jewish renaissance: the cultural revolution took place outside academia. The most cardinal phenomenon in this context - the revival of the Hebrew language - had almost no connection whatsoever with academic bodies. The phenomenon discussed in this essay should not be underestimated by historians and sociologists, especially given the fact that Israel is traditionally perceived as one of the most successful and impressive instances of nation building in the 20th century.

05-353 Kitzinger, Celia. \& Elizabeth Peel (U of York; celia_kitzinger@yahoo.com), The de-gaying and re-gaying of AIDS: contested homophobias in lesbian and gay awareness training. Discourse \& Society (London, UK) 16.2 (2005), 173-197.

From the first recognition of AIDS as a disease, it was publicly conceptualised as a 'gay plague'. In response, health education and diversity training sought to counter this association claiming that AIDS is an 'equal opportunity' virus - that it can affect anyone. In this article, the writers analyse talk about HIV/AIDS within a data corpus of thirteen tape-recorded lesbian and gay awareness training sessions. Counter to the way in which interactions are described in the lesbian and gay awareness training literature, they found that it was trainees, rather than trainers, who pursued discussions about HIV/AIDS, and who did so in order to claim the 'de-gaying' of AIDS, which they treated as representing a 'non-prejudiced' position. By contrast, and in response to trainees' insistence on de-gaying AIDS, trainers were 're-gaying' AIDS. The analysis highlights that in these sessions - designed explicitly to counter homophobic attitudes - apparently 'factual' claims and counter-claims about infection rates and risk groups are underpinned by essentially contested definitions of what constitutes a 'homophobic' attitude. The paper concludes by pointing to the value of detailed analysis of talk-ininteraction for understanding professional practices, and suggests strategies for improving the pedagogic value of training.
05-354 Lee, Seung-Hee (U of California, USA; shlee@humnet.ucla.edu), The scales of justice: balancing neutrality and efficiency in plea-bargaining encounters. Discourse \& Society (London, UK) 16.1 (2005), 33-54.

This article examines how the judge balances the normative obligation of neutrality with the bureaucratic demand for efficiency in recorded plea-bargaining encounters. The analysis demonstrates three kinds of judicial conduct by employing the methodology of conversation analysis. First, the judge displays an attitude or an outlook toward the accused or aspects of the case in an embedded manner. Second, the judge facilitates the bargaining process. The judge organises an opening and a closing of the bargaining encounters, and elicits bargaining activities from the attorneys. Third, the judge moves the bargainers toward resolution. The judge may overtly suggest a bargaining proposal, subtly intervene in the bargaining positions to show approval or disapproval, and press the bargainers to overcome obstacles. The analysis shows that the judge's conduct influences the bargaining processes and outcomes. The implication of this article is twofold. On the one hand, justice in plea bargaining is implemented in practice and is shaped by the practical ways in which judges manage their role in the interactions. On the other hand, the judge's role is shaped by the normative obligation of neutrality as reconciled with the practical demands for efficiency. Therefore, the administration of justice in plea bargaining both shapes and is shaped by the judge's conduct as balanced between neutrality and efficiency.

\section{5-355 Morales-López, Esperanza, Gabriela Prego-Vázquez \& Luzia Domínguez-Seco ( $U$ of Coruña, Spain; Ixmlopez@udc.es), Interviews between employees and customers during a company restructuring process. Discourse \& Society (London, UK) 16.2 (2005), 225-268.}

This article is the result of discursive research carried out at a company involved in a restructuring programme aimed at implementing a series of changes following the awarding of a new contract to manage a city's water supply and sewerage system. The confusion resulting from the change in management from a public to a semi-private enterprise meant employees had to deal with large numbers of complaints on a daily basis. The employees had not received any prior communicative training in order to help them resolve these situations. The authors' empirical (audio-taped) data consist of sixteen interviews between company employees and citizens analysed using methodology based on Interactional Sociolinguistics, Ethnography of Communication, and Critical Discourse Analysis. Analysis of all the interviews reveals that employees use either personalising or depersonalising strategies in order to mitigate conflicts. The analysis shows that the use of these strategies is not always appropriate, and that their success or failure depends on the types of customers and their discursive resources. 
05-356 Nero, Shondel J. (St. John's U, USA; neros@stjohns.edu), Language, identities, and ESL pedagogy. Language and Education (Clevedon, UK) 19.3 (2005), 194-211.

The multifaceted ways in which English Language Learners (ELLs) engage in 'acts of identity' through their language use have created new challenges for ESL pedagogy. Heterogeneous identities have called into question the native speaker construct and the assumed links between ethnicity and language expertise. Drawing on the research of identity theorists along with samples of completed student questionnaires, this paper argues that new paradigms are needed in second-language acquisition (SLA) theory, linguistic categorisation of students, and teacher preparation for ESL pedagogy. A pedagogical framework based on Language Identity, Awareness, and Development (LIAD) is proposed as one way of expanding on current language acquisition models.

\section{5-357 Ryoo, Hye-Kyung (Seoul Women's U; hryoo2002@yahoo.com), Achieving friendly interactions: a study of service encounters between Korean shopkeepers and African-American customers. Discourse \& Society (London, UK) 16.1 (2005), 79-105.}

This study presents an in-depth analysis of talk between Korean immigrant shopkeepers and their African-American customers in service encounters. The data were collected through nine months of fieldwork in Korean immigrant-owned stores. Despite the widely publicised conflict and tension between African Americans and Korean immigrants in service encounters, the results of this study reveal frequent incidents of positive and harmonious encounters between the participants in the stores. The study argues that a disproportionate focus toward the negative and conflictive nature of African American-Korean interactions is evident in existing research in the field. It claims that there exists an equally positive aspect of interactions between African-American customers and Korean shopkeepers during service encounter interactions that is obscured by prevailing research. This article focuses on describing this positive and friendly aspect of interactions realised through the participants' use of specific rapport-building strategies.

\section{5-358 Schoonen, Rob \& René Appel (U of Amsterdam, the Netherlands), The street language: a multilingual youth register in the Netherlands. Journal of Multilingual and Multicultural Development (Clevedon, UK) 26.2 (2005), 85-117.}

This paper reports on an empirical study of street language, a multilingual youth register in the Netherlands. Nearly 300 secondary school students completed a questionnaire on their acquaintance with and use of street language. A sub-sample of students was also inter- viewed. Use of street language will be related to background information about the students, their Dutch language proficiency and contextual characteristics. The results of the study show that the use of street language is generally widespread, but that Surinamese students are more frequent users of street language than students from other ethnic groups. Although the questionnaire did not reveal significant differences between boys and girls, the interviews showed that boys tend to use this register more intensively than girls. Contrary to popular expectations, use of street language cannot be considered to be a symptom of weak language proficiency, as users and non-users turned out to be equally proficient. The examples of street language that were provided by the students showed a high degree of influence by the Surinamese Creole, Sranan.

\section{5-359 Vari-Bogiri, Harrah ( $U$ of the South} Pacific, Port Vila, Vanuatu), A sociolinguistic survey of Araki: a dying language of Vanuatu. Journal of Multilingual and Multicultural Development (Clevedon, UK) 26.1 (2005), 52-66.

Araki is one of around a hundred languages of the Republic of Vanuatu and is spoken by the people of Araki in the north of Vanuatu. This study presents evidence to show that Araki is a declining language and adopts a diagnostic approach to finding out the underlying causes underpinning this gradual decline. This paper discusses why so many people of Araki within the younger generation have a passive knowledge of Araki while Tangoa, a closely related language, plays a dominant role in their daily communication. The focus of this survey is to expose the reasons underpinning this language change and the Araki-speaking community's attitude towards this change. With reference to other models of language revival strategies in other countries, this study proposes a model that can be employed to revive Araki. This study was carried out in 1998, using multiple data-collecting methods which comprised a questionnaire, interviews and observation of language use in various contexts, covering all the five extended families that make up the population of Arak, which stood at 121.

\section{Applied linguistics}

doi:10.1017/S026144480528299X

05-360 Chiswick, Barry, R. \& Paul W. Miller ( $U$ of Illinois at Chicago, USA), Linguistic distance: a quantitative measure of the distance between English and other languages. Journal of Multilingual and Multicultural Development (Clevedon, UK) 26.1 (2005), 1-11.

This paper develops a scalar or quantitative measure of the 'distance' between English and a myriad of other (non-native American) languages. This measure is based on the difficulty Americans have learning other languages. The linguistic distance measure is then used in an analysis of the determinants of English language 
proficiency among adult immigrants in the USA and Canada. It is shown that, when other determinants of English language proficiency are the same, the greater the measure of linguistic distance, the poorer is the respondent's English language proficiency. This measure can be used in research, evaluation and practitioner analyses, and for diagnostic purposes regarding linguistic minorities in English-speaking countries. The methodology can also be applied to develop linguistic distance measures for other languages.

05-361 Csomay, Eniko (San Diego State U, USA; ecsomay@mail.sdsu.edu), Linguistic variation within university classroom talk: a corpus-based perspective. Linguistics and Education (Amsterdam, the Netherlands) 15.3 (2005), 243-274.

The primary goal of this study is to identify intra-textual linguistic variation in university classroom talk, applying corpus-based techniques to the analysis. First, based on the automatically identified vocabulary patterns in classroom talk a large number of class sessions are segmented into smaller units of analysis. Second, the co-occurring patterns of a range of linguistic features are identified in these units applying multi-dimensional analytical techniques. Relying on these patterns, three dimensions of linguistic variation are identified in university classroom talk: contextual, directive orientation versus conceptual, informative focus; personalised framing versus lack of personalised framing; interactive dialogue versus teacher monologue. Finally, taking these dimensions, session initial differences in the discourse patterns in six disciplines and three instructional levels are discussed.

05-362 De Jong, Nel (Amsterdam U, the Netherlands; C.A.M.deJong@uva.nl), Can second language grammar be learned through listening? An experimental study. Studies in Second Language Acquisition (Cambridge, UK) 27.2 (2005), 205-234.

This study examines whether aural processing of input in a situation of implicit instruction can build a knowledge base that is available for both comprehension and production tasks. Fifty-five Dutch students learned a miniature linguistic system based on Spanish. Three training conditions were compared in which nounadjective gender agreement was the learning target. The first group of participants received receptive training, the second group received receptive and productive training, and a third group served as a control. The control group received no training of the target structure and only read an explanation of the target structure rule. Receptive knowledge was assessed with a self-paced listening test, a match-mismatch test, and a grammaticality judgment task. Productive knowledge was tested with a picture description task in single- and dual-task conditions. A post-experimental questionnaire tested whether any explicit knowledge had been induced. Results suggest that the receptive and receptive + productive training programs succeeded in building a knowledge base that was used in comprehension but much less so in production. These results are interpreted in light of processing and the distinction between implicit and explicit knowledge.

\section{5-363 Ellis, Nick C. (Michigan U, USA;} ncellis@umich.edu), At the interface: dynamic interactions of explicit and implicit language knowledge. Studies in Second Language Acquisition (Cambridge, UK) 27.2 (2005), 305-352.

This paper considers how implicit and explicit knowledge are dissociable but cooperative. It reviews various psychological and neurobiological processes by which explicit knowledge of form-meaning associations impacts upon implicit language learning. The primary conscious involvement in SLA (second language acquisition) is the explicit learning involved in the initial registration of pattern recognisers for constructions that are then tuned and integrated into the system by implicit learning during subsequent input processing. Explicit memories can also guide the conscious building of novel linguistic utterances through processes of analogy. Formulas, slot-and-frame patterns, drills and declarative pedagogical grammar rules all contribute to the conscious creation of utterances whose subsequent usage promotes implicit learning and proceduralisation. Flawed output can prompt focused feedback by way of recasts that present learners with psycholinguistic data ready for explicit analysis. Other processes of acquisition from output include differentiation, analysis and preemption. These processes of conscious construction in working memory underpin relationships between individual differences in working memory capacities and language learning aptitude.

\section{5-364 Ellis, Rod (Auckland U, New Zealand;} r.ellis@auckland.ac.nz), Measuring implicit and explicit knowledge of a second language: a psychometric study. Studies in Second Language Acquisition (Cambridge, UK) 27.2 (2005), 141-172.

A problem facing investigations of implicit and explicit learning is the lack of valid measures of second language implicit and explicit knowledge. This paper attempts to establish operational definitions of these two constructs and reports a psychometric study of a battery of tests designed to provide relatively independent measures of them. These tests were (a) an oral imitation test involving grammatical and ungrammatical sentences, (b) an oral narration test, (c) a timed grammaticality judgment test (GJT), (d) an untimed GJT with the same content, and (e) a metalinguistic knowledge test. Tests (a), (b) and (c) were designed as measures of implicit knowledge, and tests (d) and (e) were designed as measures of explicit knowledge. All of the tests examined 17 English grammatical structures. A principal 
component factor analysis produced two clear factors. This analysis showed that the scores from tests (a), (b), and (c) loaded on Factor 1, whereas the scores from ungrammatical sentences in test (d) and total scores from test (e) loaded on Factor 2. These two factors are interpreted as corresponding to implicit and explicit knowledge, respectively. A number of secondary analyses to support this interpretation of the construct validity of the tests are also reported.

\section{5-365 Handley, Zöe (U of Manchester, UK) \& Marie-Josée Hamel, Establishing a methodology for benchmarking speech synthesis for computer-assisted language learning (CALL). Language Learning \& Technology} (U of Hawaii, Manoa, USA) 9.3 (2005), 99-120.

Despite the new possibilities that speech synthesis brings about, few computer-assisted language learning (CALL) applications integrating speech synthesis have found their way onto the market. One potential reason is that the suitability and benefits of the use of speech synthesis in CALL have not been proven. One way to do this is through evaluation. Yet, very few formal evaluations of speech synthesis for CALL purposes have been conducted. One possible reason for the neglect of evaluation in this context is the fact that it is expensive in terms of time and resources. An important concern given that there are several levels of evaluation from which such applications would benefit. Benchmarking, the comparison of the score obtained by a system with that obtained by one which is known, to guarantee user satisfaction in a standard task or set of tasks, is introduced as a potential solution to this problem. In this article, we report on our progress towards the development of one of these benchmarks, namely a benchmark for determining the adequacy of speech synthesis systems for use in CALL. We do so by presenting the results of a case study which aimed to identify the criteria which determine the adequacy of the output of speech synthesis systems for use in its various roles in CALL with a view to the selection of benchmark tests which will address these criteria. These roles (reading machine, pronunciation model and conversational partner) are also discussed here. An agenda for further research and evaluation is proposed in the conclusion.

\section{5-366 Loewen, Shawn (Auckland U, New Zealand; s.loewen@auckland.ac.nz), Incidental focus on form and second language learning Studies in Second Language Acquisition (Cambridge, UK) 27.3 (2005), 361-386.}

Incidental focus on form overtly draws learners' attention to linguistic items as they arise spontaneously without prior planning - in meaning-focused interaction. This study examined the effectiveness of incidental focus on form in promoting second language (L2) learning. Seventeen hours of naturally occurring, meaning-focused L2 lessons were observed in 12 different classes of young adults in a private language school in Auckland, New Zealand. A total of 491 focus-on-form episodes (FFEs) were identified and used as a basis for individualised test items in which participants who participated in specific FFEs were asked to recall the linguistic information provided in them. The results revealed that learners were able to recall the targeted linguistic information correctly or partially correctly nearly $60 \%$ of the time one day after the FFE, and $50 \%$ of the time two weeks later. Furthermore, successful uptake in a FFE was found to be a significant predictor of correct test scores. These results suggest that incidental focus on form might be beneficial to learners, particularly if they incorporate the targeted linguistic items into their own production.

\section{5-367 Mosavi Miangah, Tayebeh \& Ali}

Delavar Khalafi (Shahre Kord U, Iran; mousavi-t@lit.sku.ac.ir), Word sense

disambiguation using target language corpus in a machine translation system. Literary and Linguistic Computing (Oxford, UK) 20.2 (2005), 237-249.

This article looks at different aspects of a new approach to word sense disambiguation using statistical information gained from a monolingual corpus of the target language. Here, the source language is English and the target is Persian. The article explains how the disambiguation method can be directly applied in the system of English-to-Persian machine translation for solving lexical ambiguity problems in this system. It explains how, unlike other disambiguation approaches using corpora for handling the problem and which use the 'Most Likelihood Model' in their statistical works, this article proposes the Random Numbers Model. It is suggested that this model is preferable from the scientific point of view as it offers the most precise and accurate results. The article outlines how this method has been tested for a selected set of English texts containing multiple-meaning words with respect to Persian language with encouraging results.

05-368 Rydberg-Cox, Jeff (U of Missouri, USA; rydbergcoxj@umkc.edu), Talking about violence: clustered participles in the speeches of lysias. Literary and Linguistic Computing (Oxford, UK) 20.2 (2005), 219-235.

This article explores the Greek participle and its use in the works of Lysias. The author argues that in Lysias' works, narrative descriptions of violence are characterised by the unusually frequent use of the participle. The article show that the association of high participle density and narratives about violence are a subset of a larger pattern relating to use of the participle in Lysias' works. In this pattern, Lysias uses unusually large numbers of participles: (1) only within the narrative and 
argumentative sections of the speeches; (2) to structure the work and mark the conclusion of narrative arcs and lines of argument; (3) in their role as a structuring device, these passages also provide immediacy and momentum to the argument or narrative descriptions of events; and (4) to mark a return in subject matter to the case at hand and to focus the attention of the jury on the question that is before them.

05-369 Tokowicz, Natasha (Pittsburgh U, USA; macw@mac.com) \& Brian MacWhinney, Implicit and explicit measures of sensitivity to violations in second language grammar: an event-related potential investigation. Studies in

Second Language Acquisition (Cambridge, UK) 27.2 (2005), 173-204.

In this study event-related brain potentials (ERPs) were used to investigate the contributions of explicit and implicit processes during second language (L2) sentence comprehension. An L2 grammaticality judgment task (GJT) was administered to twenty native English speakers enrolled in the first four semesters of Spanish. The experimental sentences varied the form of three different syntactic constructions: (a) tense-marking, which is formed similarly in the first language (L1) and the L2; (b) determiner number agreement, which is formed differently in the L1 and the L2; and (c) determiner gender agreement, which is unique to the L2. The learners were sensitive (i.e. showed different brain responses to grammatical and ungrammatical sentences) to violations in L2 for constructions that are formed similarly in the L1 and the L2, but were not sensitive to violations for constructions that differ in the L1 and the L2. Critically, a robust grammaticality effect was found in the ERP data for the construction that was unique to the L2. These findings suggest that learners are able to implicitly process some aspects of L2 syntax even in early stages of learning but that this knowledge depends on the similarity between the L1 and the L2. Comparing ERP and behavioural data could provide a sensitive method for measuring implicit processing.

05-370 Van De Mieroop, Dorien (U of Antwerp, Belgium; dorien.vandemieroop@ua.ac.be), An integrated approach of quantitative and qualitative analysis in the study of identity in speeches. Discourse \& Society (London, UK) 16.1 (2005), 107-130.

Identity is usually analysed by focusing on a single case and scrutinizing relevant text fragments. This approach rules out the possibility of doing research on larger corpora, such as this corpus of forty speeches. To this end, an integrated approach of both quantitative and qualitative analysis is proposed here. For the quantitative method, pronouns were selected as markers of the three main types of identity in the corpus, namely that of the speaker, the audience and the company the speaker represents, the latter being the focus of this article. This institutional identity construction of the company has the highest frequency in the corpus. In order to get an in-depth insight into the strategies used to build this institutional identity, extremely institutional speeches were selected and qualitative analysis was applied to this sub-corpus. This showed that three main techniques were present here, namely identification, company presentation and the suggestion of excellence.

05-371 Yamaguchi, Masataka (U of Georgia, USA; myamaguc@uga.edu), Discursive representation and enactment of national identities: the case of Generation 1.5 Japanese. Discourse \& Society (London, UK) 16.2 (2005), 269-299.

This study, as part of a larger ethnographic project, focuses on the language-in-use, in research interviews, of two Generation 1.5 Japanese, who immigrated to the US in their early life stages. To address the issue of construction of national identities, the writer examines linguistic resources that the participants use to (co-)construct their identities and stances toward the Japanese and the Americans. By drawing on discourse analysis from a multidisciplinary perspective, it has been found that the interview data contain a large number of 'complaint sequences', in which the Japanese participants complain about Japan/Japanese to a Japanese researcher; both participants represent and enact the past self in Japan negatively and the present self in the US positively; the participants take highly negative stances toward Japanese, although paradoxically identifying themselves with Japanese. The paper discusses the implications of the study for larger social processes that reconsider the complex relationships among language, ethnicities and national identity in late modernity. 